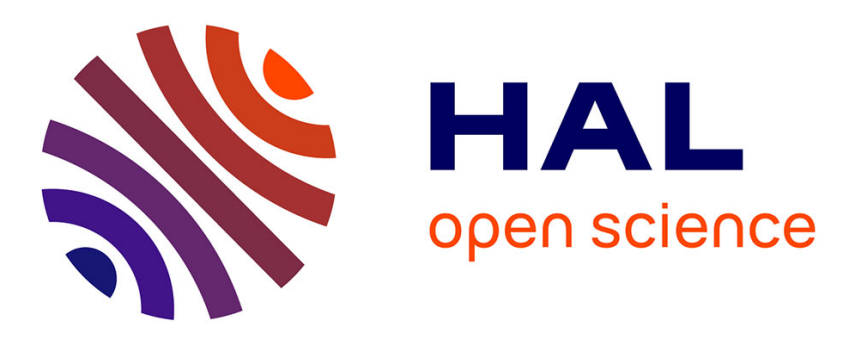

\title{
Calibration of POD reduced-order models using Tikhonov regularization
}

\author{
Laurent Cordier, Badr Abou El Majd, Julien Favier
}

\section{To cite this version:}

Laurent Cordier, Badr Abou El Majd, Julien Favier. Calibration of POD reduced-order models using Tikhonov regularization. International Journal for Numerical Methods in Fluids, 2009, 63 (2), pp.269296. 10.1002/fld.2074 . hal-01073978

\section{HAL Id: hal-01073978 https://hal.science/hal-01073978}

Submitted on 11 Oct 2014

HAL is a multi-disciplinary open access archive for the deposit and dissemination of scientific research documents, whether they are published or not. The documents may come from teaching and research institutions in France or abroad, or from public or private research centers.
L'archive ouverte pluridisciplinaire HAL, est destinée au dépôt et à la diffusion de documents scientifiques de niveau recherche, publiés ou non, émanant des établissements d'enseignement et de recherche français ou étrangers, des laboratoires publics ou privés. 


\title{
Calibration of POD Reduced-Order Models using Tikhonov regularization
}

\author{
L. Cordier ${ }^{1, *}$, B. Abou El Majd ${ }^{1}$ and J. Favier ${ }^{2}$ \\ ${ }^{1}$ LEA - CEAT, 43, rue de l'Aérodrome, 86036 Poitiers Cedex, France \\ 2 DICAT, University of Genova, via Montallegro 1, 16145 Genova, Italy
}

\begin{abstract}
SUMMARY
In this paper we compare various methods of calibration that can be used in practice to improve the accuracy of reduced-order models based on Proper Orthogonal Decomposition. The bench mark configuration retained corresponds to a case of relatively simple dynamics: a two-dimensional flow around a cylinder for a Reynolds number of 200. We generalize to the first and second-order the method of calibration based on Tikhonov regularization recently used in [1]. Finally, we show that for this flow configuration this procedure is the most effective in terms of reduction of errors.
\end{abstract}

KEY WORDS: POD Reduced Order Model ; Calibration ; Tikhonov regularization ; Optimization

\section{INTRODUCTION}

Within a relatively short period of a few years, reduced-order modeling by Proper Orthogonal Decomposition (POD) has gained a significant importance in fluid mechanics and turbulence. This sudden increase of interest is mainly linked to recent developments of technological facilities (computers, data acquisition systems) which now allow easier and faster data recording, ready-to-use for the POD (space-time correlations). However, in the majority of the applications, the studies are not carried out up to a complete and accurate representation of the flow dynamics by POD, even if the procedure is now well-known. The latter requires a Galerkin projection of the governing equations (in general the Navier-Stokes equations) onto the spatial POD functions, to obtain after truncation in the POD subspace, a set of ordinary differential equations representing the dynamics of the original system. Then, how can we explain that the most promising approaches in terms of applications: optimization and optimal control problems, real-time flow control, parametric studies, continuation methods,... are hardly used in the literature? The major barrier to the expansion of POD Galerkin approach is essentially the lack of accuracy of the reduced-order models obtained. Indeed, it is often difficult to represent with a sufficient accuracy, the dynamics at short times of the original system with

${ }^{*}$ Correspondence to: LEA - CEAT, 43, rue de l'Aérodrome, 86036 Poitiers Cedex, France. Laurent.Cordier@univ-poitiers.fr 
the parameters of control used to determine the POD basis. In these conditions, what can be expected from the ability of POD-based reduced-order models to represent the dynamics of the system at long times (bifurcation studies, for instance) or to predict the dynamics of the system for parameters of control different from those used to determine the POD basis (flow control applications)? Recently, various studies [2, 3, 4, 5, 6, 7], presenting numerical methods termed as calibration, appeared in the literature in order to improve the accuracy of POD-based reduced-order models thanks to solutions of optimization problems. The idea is simple, since the temporal dynamics of the POD model is known in advance, it is possible to use this information to correct whole or part of the coefficients issued from POD Galerkin. However, according to our knowledge of the literature, there is no trace of any comparative study of these methods. It is then difficult to claim which is the most effective method in terms of reduction of errors. The initial objective of this paper is to use a bench mark configuration, simple from the point of view of dynamics, to analyze thoroughly these various approaches, and, if possible, to release a more effective strategy. Incidentally, this approach led us to extend the method of calibration based on Tikhonov regularization [8] recently used in [1] to the first and second-order [9].

This manuscript is organized as follows. Section 2.1 introduces the Proper Orthogonal Decomposition in the general context of approximation functions. Then, the POD Galerkin approach is described for a generic controlled flow configuration (section 2.2.1). In the next subsection (section 2.2.2), the equations of the reduced-order model based on POD are simplified in order to give an overall picture of the methods of calibration. In section 2.3, the bench mark flow configuration used in this study is first described, then the necessity of introducing methods of calibration to improve the accuracy of the POD model is motivated. Section 3 is dedicated to the presentation of the existing methods of calibration, within an unified framework. The various choices of definitions of errors between the calibrated dynamics and that of the original system are first presented in section 3.1. Then, we show in section 3.2 that in the case of affine functions of errors, the minimization of the normalized errors leads to the resolution of a linear system. To conclude section 3 , the method of calibration suggested in [3] is presented in section 3.3. The various methods of calibration used so far in the literature are then compared on the bench mark configuration in section 4 . Finally, we introduce in section 5 the method of calibration based on the Tikhonov regularization, which we have developed in this paper, and present the results of a comparative study to determine the most effective method of calibration.

\section{REDUCED-ORDER MODELING BASED ON A POD GALERKIN APPROACH}

\subsection{Proper Orthogonal Decomposition}

Given a set of data, elements of a high dimensional space (potentially infinite), the main idea of the Proper Orthogonal Decomposition (POD) is to determine a subspace of reduced dimension which is optimal in the sense that the error of projection on this subspace is minimal. To formulate this statement more precisely, we introduce $H$ a Hilbert space with inner product $(\cdot, \cdot)_{H}$ and induced norm $\|\cdot\|_{H}$. In the majority of the applications, and it will be our case besides, $H$ corresponds to $L^{2}(\Omega)$, with $\Omega$ the spatial domain. Let $\mathcal{U}=\left\{\mathbf{u}\left(\mathbf{x}, t_{m}\right)=\mathbf{u}^{m}\right\}_{m=1, \ldots, N_{t}}$ be a set of $N_{t}$ snapshots taken over a time interval $[0, T]$, with 
$\mathbf{x} \in \Omega$. Then, the aim of the POD is to find a subspace $\mathcal{S}$ of dimension $N_{\text {POD }} \ll N_{t}$, such that the error $E\left(\left\|\mathbf{u}-P_{\mathcal{S}} \mathbf{u}\right\|_{H}\right)$ is minimized ${ }^{\dagger}$. Here, $E(\cdot)$ denotes an average operator over $m$, for instance an ensemble average $\left(E(\mathbf{u})=\frac{1}{N_{t}} \sum_{m=1}^{N_{t}} \mathbf{u}^{m}\right)$, and $P_{\mathcal{S}}$ the orthogonal projection onto $\mathcal{S}$. The procedure is then equivalent to minimize the expression

$$
\frac{1}{N_{t}} \sum_{m=1}^{N_{t}}\left\|\mathbf{u}^{m}-P_{\mathcal{S}} \mathbf{u}^{m}\right\|_{H}^{2}=\frac{1}{N_{t}} \sum_{m=1}^{N_{t}}\left\|\mathbf{u}^{m}-\sum_{j=1}^{N_{\mathrm{POD}}} a_{j}^{P}\left(t_{m}\right) \boldsymbol{\Phi}_{j}\right\|_{H}^{2},
$$

where $\left\{\boldsymbol{\Phi}_{j}\right\}_{j=1, \ldots, N_{\mathrm{POD}}}$ is a basis for the subspace $\mathcal{S}$ and $\left\{a_{j}^{P}\right\}_{j=1, \ldots, N_{\mathrm{POD}}}$ refer to temporal coefficients corresponding to the POD expansion (as indicated by the superscript $P$ ). It can be shown ([10] or [11] for instance) that this minimization problem leads to the eigenvalue problem:

$$
\mathcal{R} \boldsymbol{\Phi}_{j}=\lambda_{j} \boldsymbol{\Phi}_{j} \quad j=1, \cdots, N_{\mathrm{POD}},
$$

where $\mathcal{R}=E\left(\mathbf{u} \otimes \mathbf{u}^{*}\right)$. Here, $\otimes$ denotes the dyadic product between two vectors $\mathbf{u}$ and $\mathbf{u}^{*}$ where the $*$ superscript indicates complex conjugate.

Since $\mathcal{R}$ is linear, self-adjoint and positive semi-definite on $H$, the spectral theory applies and guarantees that:

1. we may choose $\boldsymbol{\Phi}_{j}$ to be orthonormal, i.e. $a_{j}^{P}\left(t_{m}\right)=\left(\mathbf{u}^{m}, \boldsymbol{\Phi}_{j}\right)$,

2. we have $\lambda_{1} \geq \lambda_{2} \geq \cdots \geq \lambda_{N_{\mathrm{POD}}} \geq 0$ where $\lambda_{j}\left(j=1, \cdots, N_{\mathrm{POD}}\right)$ are the largest $N_{\mathrm{POD}}$ eigenvalues of $\mathcal{R}$.

This approach is called direct method and corresponds to the formulation introduced originally in [12]. However, when the input data come from numerical simulations, it is much more efficient to use an alternate way of computing the POD eigenfunctions. This method, known as method of snapshots [13], consists of writing the POD modes as linear combinations of the snapshots:

$$
\boldsymbol{\Phi}_{j}(\mathbf{x})=\sum_{m=1}^{N_{t}} b_{j}^{m} \mathbf{u}^{m}(\mathbf{x}) .
$$

The vectors $\mathbf{b}_{j}=\left(b_{j}^{1}, \cdots, b_{j}^{N_{t}}\right)^{T}$ are then determined as the solutions of a new eigenvalue problem given by:

$$
\mathcal{C} \mathbf{b}_{j}=\lambda_{j} \mathbf{b}_{j},
$$

where $\mathcal{C}$ is a $N_{t} \times N_{t}$ correlation matrix with $\mathcal{C}_{i j}=\frac{1}{N_{t}}\left(\mathbf{u}^{i}, \mathbf{u}^{j}\right)$. This matrix is self-adjoint, as for the direct method. It follows that the vectors $\mathbf{b}_{j}$ are orthogonal with respect to the inner product defined by:

$$
\left(\mathbf{b}_{j}, \mathbf{b}_{k}\right)_{T}=\frac{1}{N_{t}} \sum_{m=1}^{N_{t}} b_{j}^{m} b_{k}^{m}
$$

${ }^{\dagger}$ Remind that for any orthogonal projection $P_{\mathcal{S}}$ we have $\|\mathbf{u}\|_{H}^{2}=\left\|\mathbf{u}-P_{\mathcal{S}} \mathbf{u}\right\|_{H}^{2}+\left\|P_{\mathcal{S}} \mathbf{u}\right\|_{H}^{2}$. Then, minimizing $E\left(\left\|\mathbf{u}-P_{\mathcal{S}} \mathbf{u}\right\|_{H}^{2}\right)$ is equivalent to maximizing $E\left(\left\|P_{\mathcal{S}} \mathbf{u}\right\|_{H}^{2}\right)$. 
By extension, the inner product associated with the direct method will henceforth be denoted $\left(\boldsymbol{\Phi}_{j}, \boldsymbol{\Phi}_{k}\right)_{\Omega}$ where by definition:

$$
\left(\mathbf{\Phi}_{j}, \boldsymbol{\Phi}_{k}\right)_{\Omega}=\int_{\Omega} \boldsymbol{\Phi}_{j}(\mathbf{x}) \cdot \boldsymbol{\Phi}_{k}(\mathbf{x}) d \mathbf{x}
$$

with $\cdot$ the notation of the standard Euclidean inner product.

\subsection{POD Reduced-Order Model based on Galerkin projection}

2.2.1. General description For incompressible flows, the motion of the fluid is described by the incompressibility condition and the Navier-Stokes equations,

$$
\begin{aligned}
\nabla \cdot \mathbf{u} & =0 \\
\partial_{t} \mathbf{u} & =\mathcal{N}(\mathbf{u})-\nabla p \quad \text { with } \quad \mathcal{N}(\mathbf{u})=-(\mathbf{u} \cdot \nabla) \mathbf{u}+\frac{1}{R e} \mathbf{\Delta} \mathbf{u}
\end{aligned}
$$

In these equations, all variables ( $\mathbf{u}$ velocity vector and $p$ pressure) are assumed to be nondimensional and $R e$ is the Reynolds number.

The POD Reduced-Order Model (POD ROM) is then constructed by applying the Galerkin projection to the governing equations. Since by construction the eigenfunctions $\boldsymbol{\Phi}_{i}$ are divergence-free for an incompressible flow, they can be used as test functions to derive the variational formulation of the Navier-Stokes equations:

$$
\left(\partial_{t} \mathbf{u}, \boldsymbol{\Phi}_{i}\right)_{\Omega}=\left(\mathcal{N}(\mathbf{u}), \boldsymbol{\Phi}_{i}\right)_{\Omega}-\left(\nabla p, \boldsymbol{\Phi}_{i}\right)_{\Omega}
$$

Using the relation $\nabla \cdot \boldsymbol{\Phi}_{i}=0$, the pressure term can be rewritten as a boundary term:

$$
\left(\nabla p, \boldsymbol{\Phi}_{i}\right)_{\Omega}=\int_{\Omega} \boldsymbol{\Phi}_{i} \cdot \nabla p d \mathbf{x}=\int_{\Omega} \nabla \cdot\left(p \boldsymbol{\Phi}_{i}\right) d \mathbf{x}=\int_{\partial \Omega} p \boldsymbol{\Phi}_{i} \cdot \mathbf{n} d \mathbf{x}=P_{i},
$$

where $\mathbf{n}$ is the outward unit normal at the boundary surface $\partial \Omega$. If the snapshots $\mathbf{u}^{m}$ employed for the POD are zero on the boundary, then $\boldsymbol{\Phi}_{i}=0$ on $\partial \Omega$ and the pressure term vanishes. In most of the applications $[14,2,4]$, the contribution of the pressure term is simply neglected as a first approximation.

To carry on our developments, we have to specify the variable on which the POD will be applied. Beyond the idea of a correct representation of the flow physics, we would like to simplify as much as possible the analytical expressions of the coefficients associated to the POD ROM. In the previous paragraph, we point out that prescribing $\boldsymbol{\Phi}_{i}=\mathbf{0}$ on the boundary is relevant for the pressure term. We will thus use a lifting procedure discussed thoroughly in [15]: if the boundary conditions are time-independent (uncontrolled flow for instance), the original set of snapshots $\mathcal{U}$ is replaced by $\mathcal{U}^{\prime}=\left\{\mathbf{u}^{m}-\mathbf{u}_{m}\right\}_{m=1, \ldots, N_{t}}$ where $\mathbf{u}_{m}=E(\mathbf{u})$; if the boundary conditions are time-dependent (flow controlled at the boundary for instance), the POD is applied to $\mathcal{U}^{\prime \prime}=\left\{\mathbf{u}^{m}-\gamma^{m} \mathbf{u}_{c}-\mathbf{u}_{m}\right\}_{m=1, \ldots, N_{t}}$ where $\gamma^{m}=\gamma\left(t_{m}\right)$ characterizes the time evolution of the control and $\mathbf{u}_{c}$ is the actuation mode. In practice, this mode is determined as a particular solution of the Navier-Stokes equations, where the boundary conditions on the controlled part of $\partial \Omega$ are equal to the spatial evolution of the control, and where the boundary conditions on the uncontrolled part are set to zero (see [16] for an application to the controlled cylinder wake). 
In the general formulation, the velocity expansion of $\mathbf{u}$ over the POD modes $\boldsymbol{\Phi}_{j}$ writes:

$$
\mathbf{u}(\mathbf{x}, t)=\mathbf{u}_{m}(\mathbf{x})+\gamma(t) \mathbf{u}_{c}(\mathbf{x})+\sum_{j=1}^{N_{\text {POD }}} a_{j}(t) \boldsymbol{\Phi}_{j}(\mathbf{x}) .
$$

By substituting (2) into the Galerkin projection (1), we obtain after some algebraic manipulations [17] the following expression for the reduced-order model in the presence of control:

$$
\begin{aligned}
\dot{a}_{i}^{R}(t)= & A_{i}^{G P}+B_{i j}^{G P} a_{j}^{R}(t)+C_{i j k}^{G P} a_{j}^{R}(t) a_{k}^{R}(t)-P_{i}(t) \\
& +D_{i}^{G P} \dot{\gamma}(t)+\left(E_{i}^{G P}+F_{i j}^{G P} a_{j}^{R}(t)\right) \gamma(t)+G_{i}^{G P} \gamma^{2}(t) \\
a_{i}^{R}(0)= & a_{i}^{P}(0)=\left(\mathbf{u}(\mathbf{x}, 0)-\mathbf{u}_{m}(\mathbf{x})-\gamma(0) \mathbf{u}_{c}(\mathbf{x}), \mathbf{\Phi}_{i}\right)_{\Omega},
\end{aligned}
$$

where the Einstein summation is used and all subscripts $i, j, k$ run from 1 to $N_{\text {gal }}$. Here, $N_{\text {gal }}<N_{\text {POD }}$ corresponds to the number of Galerkin modes retained in the reduced-order model. This number of modes is assumed to be sufficient to reproduce accurately the flow (see section 2.3 to evaluate $N_{\text {gal }}$ ). The coefficients $A_{i}^{G P}, B_{i j}^{G P}, C_{i j k}^{G P}, D_{i}^{G P}, E_{i}^{G P}, F_{i j}^{G P}$ and $G_{i}^{G P}$ depend explicitly on $\boldsymbol{\Phi}, \mathbf{u}_{m}$ and $\mathbf{u}_{c}$ (see Appendix I). The superscripts $R$ are introduced for the temporal coefficients $a_{i}$ are to indicate that these coefficients are obtained by integrating in time the system of ODEs (3). Similarly, we introduce the superscripts $G P$ for the coefficients of the POD ROM to stress that these coefficients are obtained directly by Galerkin projection.

2.2.2. Simplification of the POD ROM In practice, the POD ROM (3) will not necessarily be integrated in time with the coefficients determined by the method of POD Galerkin. Indeed, whole or part of the coefficients may either be unknown (in the case of experimental data for instance) or known with an insufficient level of accuracy to reproduce correctly the original dynamics (see section 2.3 for an example). It is thus necessary to identify, or in other words calibrate, whole or part of the coefficients. Since the structure of $(3 \mathrm{a})$ is not modified by the pressure term [18], we can consider without restricting the generality $P_{i}(t)=0$.

In section 3 , the various methods of calibration encountered in the literature will be presented within a unified framework. Therefore we introduce $\mathbf{a}^{R}(t)=\left(a_{1}^{R}(t), \cdots, a_{N_{\mathrm{gal}}}^{R}(t)\right)^{T}$, vectorial solution of (3), to rewrite compactly (3a) as:

$$
\begin{aligned}
\dot{a}_{i}^{R}(t) & =f_{i}\left(\mathbf{y}_{i}, \mathbf{a}^{R}(t)\right)+g_{i}\left(\mathbf{z}_{i}, \mathbf{a}^{R}(t), \gamma\right) \quad i=1, \cdots, N_{\text {gal }}, \\
\text { where } f_{i}\left(\mathbf{y}_{i}, \mathbf{a}^{R}(t)\right) & =A_{i}+B_{i j} a_{j}^{R}(t)+Q_{i j k} a_{j}^{R}(t) a_{k}^{R}(t), \\
\text { and } g_{i}\left(\mathbf{z}_{i}, \mathbf{a}^{R}(t), \gamma\right) & =D_{i} \dot{\gamma}(t)+\left(E_{i}+F_{i j} a_{j}^{R}(t)\right) \gamma(t)+G_{i} \gamma^{2}(t)
\end{aligned}
$$

with $j=1, \cdots, N_{\text {gal }}$ and $k=1, \cdots, j$. In (4a), $\mathbf{y}_{i}$ and $\mathbf{z}_{i}$ denote the unknown coefficients corresponding to respectively the uncontrolled and controlled part of (3a), i.e. 


$$
\mathbf{y}_{i}=\left(\begin{array}{c}
A_{i} \\
B_{i 1} \\
\vdots \\
B_{i N_{\text {gal }}} \\
Q_{i 11} \\
\vdots \\
Q_{i N_{\text {gal }} N_{\text {gal }}}
\end{array}\right) \in \mathbb{R}^{N_{y_{i}}} \text { and } \quad \mathbf{z}_{i}=\left(\begin{array}{c}
D_{i} \\
E_{i} \\
F_{i 1} \\
\vdots \\
F_{i N_{\text {gal }}} \\
G_{i}
\end{array}\right) \in \mathbb{R}^{N_{z_{i}}},
$$

where $N_{y_{i}}=1+N_{\text {gal }}+\frac{N_{\text {gal }}\left(N_{\text {gal }}+1\right)}{2}$ and $N_{z_{i}}=3+N_{\text {gal }}$. In (4b), the coefficients $Q_{i j k}$ correspond to the symmetric part of $C_{i j k}$ only, i.e. $Q_{i j k}=1 / 2\left(C_{i j k}+C_{i k j}\right)$ for $i, j=1, \cdots, N_{\text {gal }}$ and $k=1, \cdots, j$. This modification of the expression of the quadratic term is justified by the fact that it is impossible to differentiate $C_{i j k}$ from $C_{i k j}$ by any technique of calibration.

In vectorial formulation, the controlled POD ROM given by (4a) is finally:

$$
\dot{\mathbf{a}}^{R}(t)=\mathbf{f}\left(\mathbf{y}, \mathbf{a}^{R}(t)\right)+\mathbf{g}\left(\mathbf{z}, \mathbf{a}^{R}(t), \gamma\right),
$$

where for the uncontrolled contribution:

$$
\mathbf{f}=\left(\begin{array}{c}
f_{1} \\
f_{2} \\
\vdots \\
f_{N_{\text {gal }}}
\end{array}\right) \in \mathbb{R}^{N_{\text {gal }}} \quad ; \quad \mathbf{y}=\left(\begin{array}{c}
\mathbf{y}_{1} \\
\mathbf{y}_{2} \\
\vdots \\
\mathbf{y}_{N_{\text {gal }}}
\end{array}\right) \in \mathbb{R}^{N_{\mathbf{y}}} \quad \text { with } \quad N_{\mathbf{y}}=N_{\text {gal }} N_{y_{i}},
$$

and, for the controlled contribution:

$$
\mathbf{g}=\left(\begin{array}{c}
g_{1} \\
g_{2} \\
\vdots \\
g_{N_{\text {gal }}}
\end{array}\right) \in \mathbb{R}^{N_{\text {gal }}} \quad ; \quad \mathbf{z}=\left(\begin{array}{c}
\mathbf{z}_{1} \\
\mathbf{z}_{2} \\
\vdots \\
\mathbf{z}_{N_{\text {gal }}}
\end{array}\right) \in \mathbb{R}^{N_{\mathbf{z}}} \quad \text { with } \quad N_{\mathbf{z}}=N_{\text {gal }} N_{z_{i}} .
$$

Here, $\mathbf{f}$ and $\mathbf{g}$ can be evaluated easily using the coefficients of the model $\mathbf{y}$ and $\mathbf{z}$ (see Appendix II).

For the sake of clarity, the rest of the paper is limited to uncontrolled flows, i.e. $\gamma=0$ or $\mathbf{g}=\mathbf{0}$, in (5). One of the interests of (5) is to clearly demonstrate that for a given value of $\gamma$ the extension to controlled flows is straightforward (see [1] for a recent application of the calibration techniques to a controlled wake flow).

\subsection{Two-dimensional cylinder wake flow at $R e=200$}

The reduced-order modeling approach based on POD is now applied to a two-dimensional incompressible cylinder wake flow at $R e=200$. The database was computed using a finiteelement code (DNS code Icare, IMFT/University of Toulouse, see [19] for details) and contains $N_{t}=200$ two-dimensional snapshots of the flow velocity, taken over a period $T=12$ i.e. over more than two periods of vortex shedding $\left(T_{\mathrm{vs}}=5\right)$. Typical iso-values of the longitudinal 


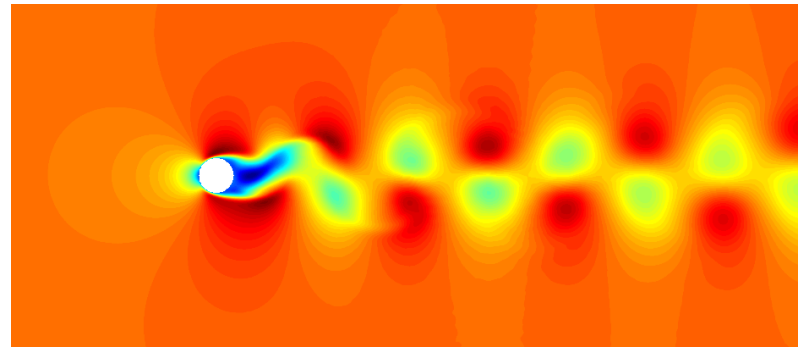

Figure 1. Iso-values of the longitudinal velocity fluctuation.

velocity are shown in Fig. 1.

Following the discussion of section 2.2.1, the method of snapshots introduced in section 2.1 is applied to the velocity fluctuation. The first six spatial eigenfunctions $\boldsymbol{\Phi}_{i}$ are represented in Fig. 2 and the relative kinetic energy is plotted in logarithmic scale for the first 40 POD modes in Fig. 3. The energy is clearly concentrated in a very small number of modes: the first six POD modes are sufficient to represent $99.9 \%$ of the flow energy and we thus consider $N_{\text {gal }}=6$ to derive the POD ROM.

The POD ROM (5) is then integrated in time with a classical fourth-order Runge-Kutta scheme and a time step of $10^{-3} T$. A set of predicted time histories for the mode amplitudes $a_{i}^{R}(t)$ is obtained, and compared to the set of POD temporal eigenfunctions $a_{i}^{P}(t)$. As shown in Fig. 4, the original dynamics is globally well reproduced but the accuracy is not perfect. Indeed, the most energetic mode is well reconstructed, while for the higher modes the maxima remain over-estimated. Besides that, a constant phase shift can quickly occur between the dynamics of the model and that of the original one (see mode 3 for instance). If the final objective is to use the POD ROM in a phase control strategy, then these errors can lead to the failure of this control strategy. This relatively bad performances of the model may be attributed to

i) the structural instability of the Galerkin projection [20, 21, 14],

ii) the truncation of the POD basis (dissipative scales associated to higher POD modes are not sufficiently present in the model),

iii) the fact that the set of data does not perfectly respect the weak formulation (5): inaccurate treatment of the boundary terms and the pressure contribution, incompressibility not verified (experimental data),

$i v$ ) an insufficient numerical precision to compute the POD ROM coefficients.

It is thus necessary to introduce calibration techniques in order to reproduce accurately the dynamics of reference using the POD ROM. 


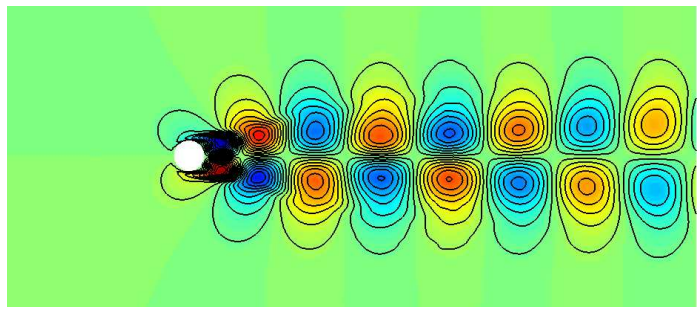

(a) Mode 1 .

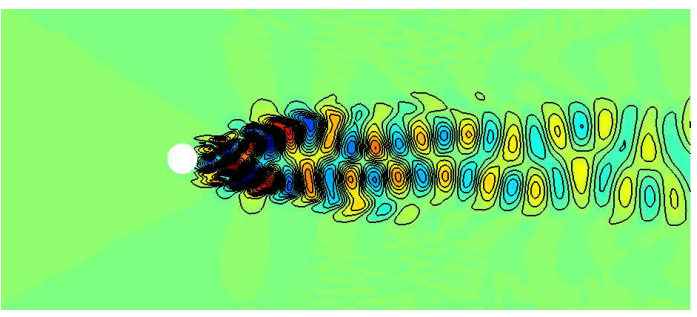

(c) Mode 3 .

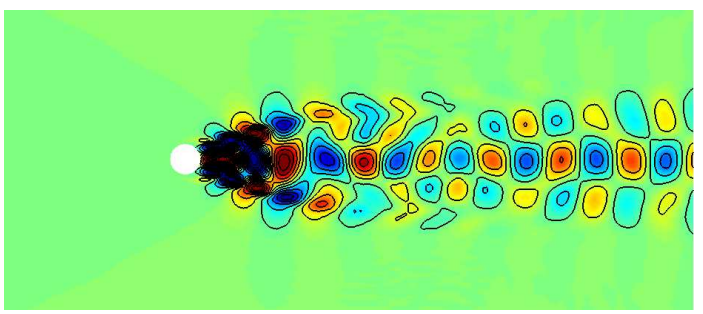

(e) Mode 5 .

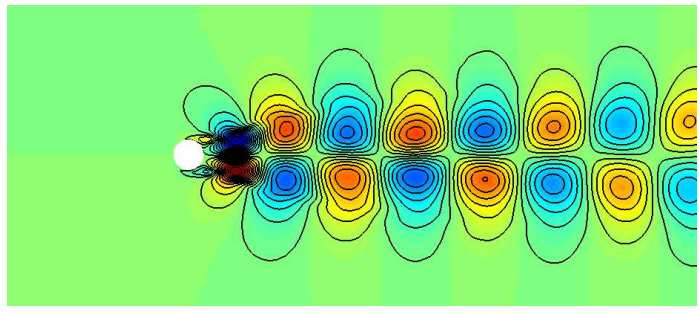

(b) Mode 2 .

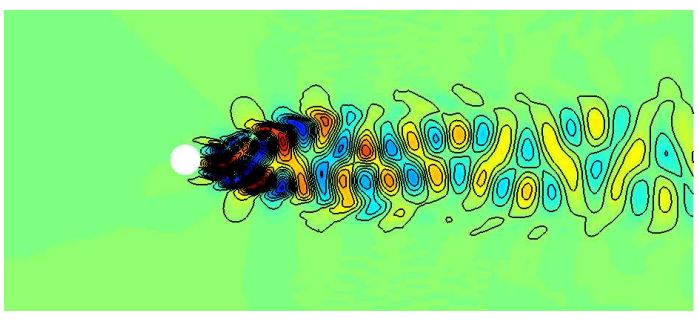

(d) Mode 4 .

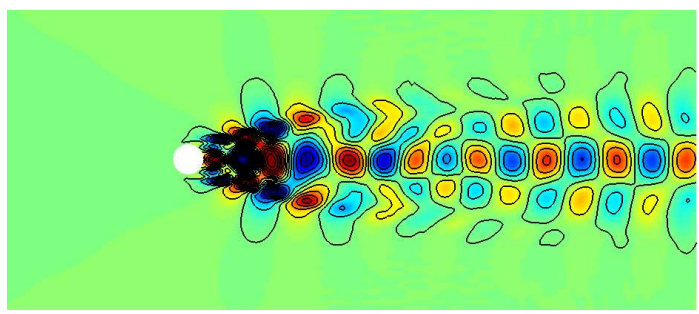

(f) Mode 6 .

Figure 2. The first 6 spatial POD eigenfunctions are visualized by iso-values of their norm $\left(\left\|\boldsymbol{\Phi}_{i}\right\|_{\Omega}\right)$.

\section{EXISTING CALIBRATION METHODS}

Many methods of calibration have already been proposed in the literature. Let us quote for example the different techniques based on least-square minimization $[2,3,5]$, those consisting in solving a constrained optimization problem, iteratively [22] or simultaneously [6], the recent method termed intrinsic stabilization introduced in [7], and finally the calibration procedure suggested by [3]. To compare fairly the previous procedures on the case of the cylinder wake flow (see section 4), we first introduce various criteria of error (section 3.1), along the lines of [3]. Then, we emphasize in section 3.2 that the problem of minimization reduces to a linear system when the errors are affine functions of $\mathbf{y}$. Finally, the calibration procedure suggested in [3] is presented, as the Tikhonov regularization method proposed in this paper (section 5) constitutes an alternative to their approach. 


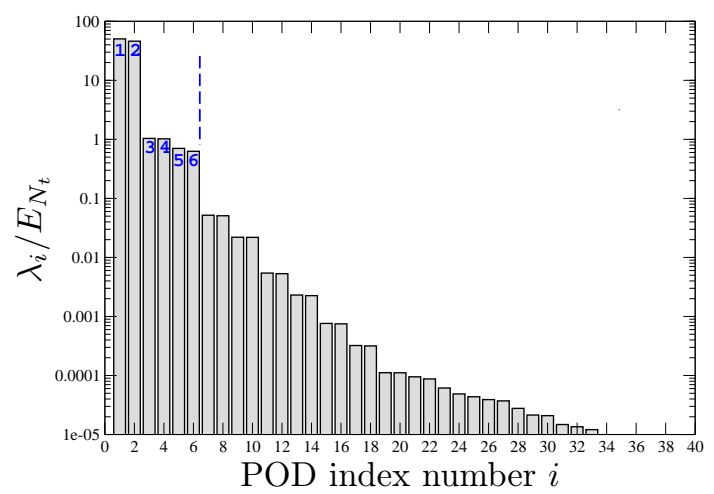

Figure 3. POD eigenvalues in logarithmic scale. $E_{N_{t}}$ corresponds to twice the energy contained in the database $\left(E_{N_{t}}=\sum_{j=1}^{N_{t}} \lambda_{j}\right)$.

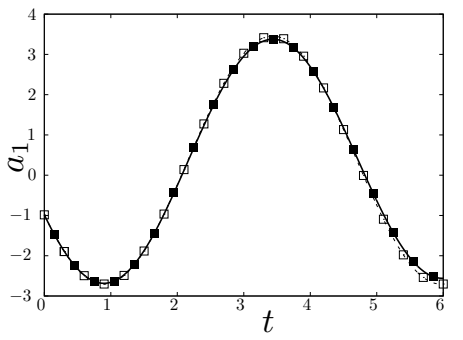

(a) Mode 1

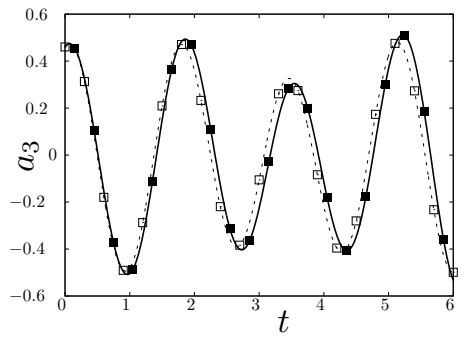

(b) Mode 3

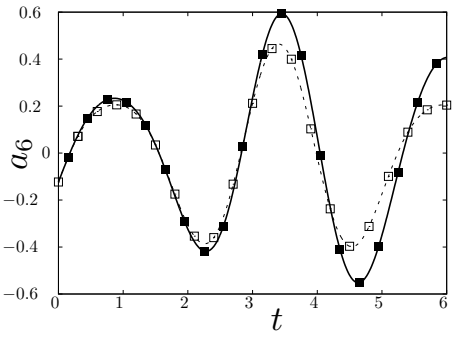

(c) Mode 6

Figure 4. Comparison between the temporal evolutions of the projected $\square$ (POD) and predicted (POD ROM) mode amplitudes. No calibration was used for the POD ROM.

\subsection{Definitions of errors}

3.1.1. State calibration method with dynamical constraints The objective of the POD-based model (5) is to represent, as accurately as possible, the dynamics given by the POD temporal eigenfunctions. It is then natural to seek the coefficients $\mathbf{y}$ which minimize the error

$$
\mathbf{e}^{(1)}(\mathbf{y}, t)=\mathbf{a}^{P}(t)-\mathbf{a}^{R}(t),
$$

under the constraint of the Cauchy problem defined by:

$$
\left(\mathcal{P}_{C}\right)\left\{\begin{array}{l}
\dot{\mathbf{a}}^{R}(t)=\mathbf{f}\left(\mathbf{y}, \mathbf{a}^{R}(t)\right), \\
\mathbf{a}^{R}(0)=\mathbf{a}^{P}(0) .
\end{array}\right.
$$

Since $\mathbf{e}^{(1)} \in \mathbb{R}^{N_{\text {gal }}}$ and is time-dependent, we rather seek to minimize

$$
\mathcal{I}^{(1)}(\mathbf{y})=\left\langle\left\|\mathbf{e}^{(1)}(\mathbf{y}, t)\right\|_{\Lambda}^{2}\right\rangle_{T_{o}},
$$


where $\langle\cdot\rangle_{T_{o}}$ is a time average operator over $\left[0, T_{o}\right]\left(T_{o} \leq T\right)$ and $\|\cdot\|_{\Lambda}$ is a norm of $\mathbb{R}^{N_{\text {gal }}} \cdot\langle\cdot\rangle_{T_{o}}$ corresponds in this paper to the arithmetic time-average on $N$ equally spaced elements of the interval $\left[0, T_{o}\right]$ :

$$
\langle g(t)\rangle_{T_{o}}=\frac{1}{N} \sum_{k=1}^{N} g\left(t_{k}\right) \quad \text { with } \quad t_{k}=(k-1) \Delta t \quad \text { and } \quad \Delta t=\frac{T_{o}}{N-1} .
$$

For the norm, we introduce $\Lambda \in \mathbb{R}^{N_{\text {gal }} \times N_{\text {gal }}}$ the symmetric definite positive matrix associated to $\|\cdot\|_{\Lambda}$ and define for any $\mathbf{e} \in \mathbb{R}^{N_{\text {gal }} \text { : }}$

$$
\|\mathbf{e}\|_{\Lambda}^{2}=\mathbf{e}^{T} \Lambda \mathbf{e}
$$

The matrix $\Lambda$ introduced in the definition acts as a weight function: it allows to balance the importance of specific POD modes. When $\Lambda=I_{N_{\text {gal }}}$ for example, all POD modes have the same importance in terms of error.

The minimization of $\mathcal{I}^{(1)}$ under the constraints of $\mathcal{P}_{C}$ corresponds to a nonlinear constrained optimization problem. Until now, this problem is solved only for $\Lambda=I_{N_{\text {gal }}}$ i.e. minimizing:

$$
\mathcal{I}^{(1)}(\mathbf{y})=\frac{1}{N} \sum_{k=1}^{N} \sum_{i=1}^{N_{\text {gal }}}\left(a_{i}^{P}\left(t_{k}\right)-a_{i}^{R}\left(t_{k}\right)\right)^{2} .
$$

In [22], the constrained optimization problem built on $\mathcal{I}^{(1)}$ was solved iteratively to find optimal eddy viscosities. In [2], the same iterative approach is used to determine a linear model for the pressure contribution of the Galerkin projection. More recently, a similar approach was used in [6] to determine the constant and linear coefficients of the POD ROM. However, in this case, the constrained optimization problem was solved simultaneously with a pseudo-spectral discretization of the variables.

3.1.2. State calibration method From a mathematical point of view, the minimization problem based on $\mathcal{I}^{(1)}$ is not well posed: several solutions may coexist and convergence is not even guaranteed (see [3] for some arguments). Therefore, it was proposed in [3] to suppress the dynamical constraint in the definition of $\mathbf{e}^{(1)}$.

After integration in time of the POD ROM, the error $\mathbf{e}^{(1)}$ can be rewritten as:

$$
\mathbf{e}^{(1)}(\mathbf{y}, t)=\mathbf{a}^{P}(t)-\mathbf{a}^{P}(0)-\int_{0}^{t} \mathbf{f}\left(\mathbf{y}, \mathbf{a}^{R}(\tau)\right) d \tau .
$$

To suppress the nonlinear constraint, the occurrence of $\mathbf{a}^{R}$ in the last term can be replaced by $\mathbf{a}^{P}$. We then introduce a new error $\mathbf{e}^{(2)}$ defined as:

$$
\mathbf{e}^{(2)}(\mathbf{y}, t)=\mathbf{a}^{P}(t)-\mathbf{a}^{P}(0)-\int_{0}^{t} \mathbf{f}\left(\mathbf{y}, \mathbf{a}^{P}(\tau)\right) d \tau .
$$

In the literature, the minimization of $\mathcal{I}^{(2)}(\mathbf{y})=\left\langle\left\|\mathbf{e}^{(2)}(\mathbf{y}, t)\right\|_{\Lambda}^{2}\right\rangle_{T_{o}}$ was considered in [3] to determine all the coefficients of the model and more recently in [23] to evaluate the constant and linear coefficients for an unsteady transonic flow. In both cases, all modes have the same contribution, i.e. $\Lambda=I_{N_{\text {gal }}}$, and the following error is minimized:

$$
\mathcal{I}^{(2)}(\mathbf{y})=\frac{1}{N} \sum_{k=1}^{N} \sum_{i=1}^{N_{\text {gal }}}\left(a_{i}^{P}\left(t_{k}\right)-a_{i}^{P}(0)-\int_{0}^{t_{k}} f_{i}\left(\mathbf{y}, \mathbf{a}^{P}(\tau)\right) d \tau\right)^{2} .
$$


3.1.3. Flow calibration method The third criterion of error is obtained by taking the temporal derivative of the $\mathbf{e}^{(1)}$ criterion:

$$
\frac{d}{d t}\left(\mathbf{e}^{(1)}(\mathbf{y}, t)\right)=\dot{\mathbf{a}}^{P}(t)-\mathbf{f}\left(\mathbf{y}, \mathbf{a}^{R}(t)\right)
$$

and by replacing $\mathbf{a}^{R}$ by $\mathbf{a}^{P}$ in order to suppress the nonlinear constraint. The following error is thus obtained:

$$
\mathbf{e}^{(3)}(\mathbf{y}, t)=\dot{\mathbf{a}}^{P}(t)-\mathbf{f}\left(\mathbf{y}, \mathbf{a}^{P}(t)\right) .
$$

The idea suggested by [3] to minimize $\mathcal{I}^{(3)}(\mathbf{y})=\left\langle\left\|\mathbf{e}^{(3)}(\mathbf{y}, t)\right\|_{\Lambda}^{2}\right\rangle_{T_{o}}$ seems natural because it is equivalent to impose that the temporal POD eigenfunctions $\mathbf{a}^{P}$ are solutions of the flow model given by $\mathbf{f}$. For this reason, this least-square procedure was proposed independently by [2] to evaluate a linear model for the pressure term coming from the Galerkin projection and by [5] to identify all the coefficients of the model starting from experimental data obtained by PIV. All these applications were carried out for $\Lambda=I_{N_{\text {gal }}}$ i.e. with the aim of minimizing:

$$
\left\langle\left\|\mathbf{e}^{(3)}(\mathbf{y}, t)\right\|_{I_{N_{g a l}}}^{2}\right\rangle_{T_{o}}=\frac{1}{N} \sum_{k=1}^{N} \sum_{i=1}^{N_{\mathrm{gal}}}\left(\dot{a}_{i}^{P}\left(t_{k}\right)-f_{i}\left(\mathbf{y}, a_{i}^{P}\left(t_{k}\right)\right)^{2} .\right.
$$

\subsection{Affine functions of errors}

For the state calibration method (section 3.1.2) and the flow calibration method (section 3.1.3), the corresponding errors are affine with respect to $\mathbf{y}$. We can then demonstrate (see Appendix III) that minimizing

$$
\mathcal{I}^{(i)}(\mathbf{y})=\left\langle\left\|\mathbf{e}^{(i)}(\mathbf{y}, t)\right\|_{\Lambda}^{2}\right\rangle_{T_{o}} \quad \text { for } i=2,3
$$

gives rise to the linear system

$$
A^{(i)} \mathbf{y}=\mathbf{b}^{(i)}
$$

where $A^{(i)} \in \mathbb{R}^{N_{\mathbf{y}} \times N_{\mathbf{y}}}$ and $\mathbf{b}^{(i)} \in \mathbb{R}^{N_{\mathbf{y}}}$ are defined in Appendix III.

\subsection{Calibration procedure proposed in [3]}

The general idea is to determine the coefficients $\mathbf{y}_{\alpha}^{(i)}$, which characterize uniquely the calibrated model, as the solution of the optimization problem based on the functional:

$$
\mathcal{J}_{\alpha}^{(i)}(\mathbf{y})=(1-\alpha) \mathcal{E}^{(i)}(\mathbf{y})+\alpha \mathcal{D}(\mathbf{y}) \quad \text { with } i=2,3 .
$$

$\alpha \in[0,1]$ is a weighting parameter; $\mathcal{E}^{(i)}(\mathbf{y})$ is a measure of the normalized error between the behavior of the data i.e. $\mathbf{a}^{P}(t)$, and the behavior of the polynomial model defined by $\mathbf{y}$ whose state is $\mathbf{a}^{R}(t) ; \mathcal{D}(\mathbf{y})$ is a measure of the difference between the coefficients of the model $\mathbf{y}$ and the coefficients obtained from the Galerkin projection $\mathbf{y}^{G P}$. For $\alpha=0$, the calibrated model is fully optimized (we will see in section 4 that in this case the linear system (6) is ill-conditioned) and for $\alpha=1$, the coefficients from the Galerkin projection are recovered. $\mathcal{E}^{(i)}$ and $\mathcal{D}$ are defined as:

$$
\mathcal{E}^{(i)}(\mathbf{y})=\frac{\left\langle\left\|\mathbf{e}^{(i)}(\mathbf{y}, t)\right\|_{\Lambda}^{2}\right\rangle_{T_{o}}}{\left\langle\left\|\mathbf{e}^{(i)}\left(\mathbf{y}^{G P}, t\right)\right\|_{\Lambda}^{2}\right\rangle_{T_{o}}}=\frac{\mathcal{I}^{(i)}(\mathbf{y})}{\mathcal{I}^{(i)}\left(\mathbf{y}^{G P}\right)}
$$


and

$$
\mathcal{D}(\mathbf{y})=\frac{\left\|\mathbf{y}-\mathbf{y}^{G P}\right\|_{\Pi}^{2}}{\left\|\mathbf{y}^{G P}\right\|_{\Pi}^{2}}
$$

where $\|\cdot\|_{\Pi}$ is a semi-norm on the polynomial vector space. For any $\mathbf{y} \in \mathbb{R}^{N_{\mathbf{y}}},\|\mathbf{y}\|_{\Pi}$ is defined by

$$
\|\mathbf{y}\|_{\Pi}^{2}=\mathbf{y}^{T} \Pi \mathbf{y}
$$

where $\Pi \in \mathbb{R}^{N_{\mathbf{y}} \times N_{\mathbf{y}}}$ is a non-negative symmetric matrix. For $\Pi=I_{N_{\mathbf{y}}}$, all the coefficients are calibrated and have the same weight in the calibration. For values different from $I_{N_{\mathrm{y}}}$, a partial calibration is possible (see [3] for the only example present in the literature). In section 4, our numerical experiments will be carried out for $\Pi=I_{N_{\mathbf{y}}}$.

Finally, it can be shown (Appendix IV) that for $i=2$ or 3 , the minimization of $\mathcal{J}_{\alpha}^{(i)}$ amounts to solve the linear system:

$$
A_{\alpha}^{(i)} \mathbf{y}=\mathbf{b}_{\alpha}^{(i)}
$$

with

$$
A_{\alpha}^{(i)}=\frac{1-\alpha}{\mathcal{I}^{(i)}\left(\mathbf{y}^{G P}\right)} A^{(i)}+\frac{\alpha}{\left\|\mathbf{y}^{G P}\right\|_{\Pi}^{2}} \Pi
$$

and

$$
\mathbf{b}_{\alpha}^{(i)}=\frac{1-\alpha}{\mathcal{I}^{(i)}\left(\mathbf{y}^{G P}\right)} \mathbf{b}^{(i)}+\frac{\alpha}{\left\|\mathbf{y}^{G P}\right\|_{\Pi}^{2}} \Pi \mathbf{y}^{G P} .
$$

The questions which remain open are:

1. How to fix the weighting parameter $\alpha$, and what is the optimal choice?

2. How to apply this procedure of calibration in the case of experimental data, for which the coefficients coming from the Galerkin projection are usually not available?

Table I. Normalized errors $\mathcal{E}^{(i)}$ and costs of the calibration $\mathcal{D}$. Comparison between the results obtained by: $i$ ) minimizing $\mathcal{I}^{(1)}$ under the constraint of $\left.\mathcal{P}_{C}, i i\right)$ minimizing $\mathcal{I}^{(3)}$ with the determinations of the linear $(L)$, constant and linear $(C$ and $L)$ and eddy-viscosity terms $(V)$ and $i i i)$ Intrinsic Stabilization $[7]$

\begin{tabular}{|l|c|c|c|c||c|}
\hline Method of calibration & Control terms & $\sqrt{\mathcal{E}^{(1)}(\mathbf{y})}$ & $\sqrt{\mathcal{E}^{(2)}(\mathbf{y})}$ & $\sqrt{\mathcal{E}^{(3)}(\mathbf{y})}$ & $\sqrt{\mathcal{D}(\mathbf{y})}$ \\
\hline Minimization of $\mathcal{I}^{(1)}$ & $C$ and $L$ & $2.6010^{-2}$ & $2.4310^{-1}$ & $2.4410^{-1}$ & $1.5310^{-1}$ \\
under the constraint of $\mathcal{P}_{C}$ & & & & & \\
Minimization of $\mathcal{I}^{(3)}$ & $L$ & $6.7810^{-2}$ & $8.8510^{-1}$ & $3.8610^{-1}$ & $3.2810^{-2}$ \\
Minimization of $\mathcal{I}^{(3)}$ & $C$ and $L$ & $3.1910^{-2}$ & $2.4610^{-1}$ & $2.6810^{-1}$ & $3.2710^{-2}$ \\
Minimization of $\mathcal{I}^{(3)}$ & $V$ & $7.8510^{-1}$ & $8.7210^{-1}$ & $9.1810^{-1}$ & $1.1910^{-2}$ \\
Intrinsic Stabilization [7] & $C$ and $L$ & $6.2610^{-2}$ & $3.2910^{-1}$ & $3.0710^{-1}$ & $3.2710^{-2}$ \\
\hline
\end{tabular}




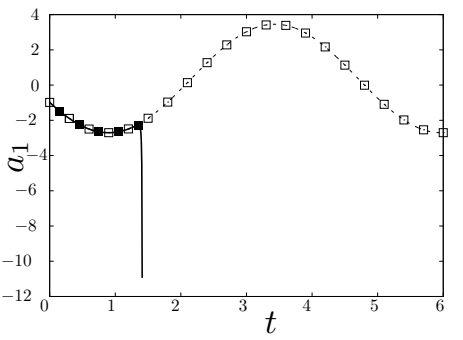

(a) Mode 1

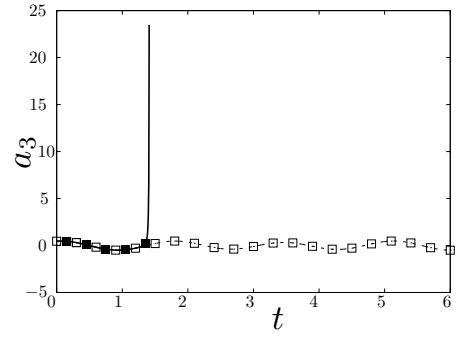

(b) Mode 3

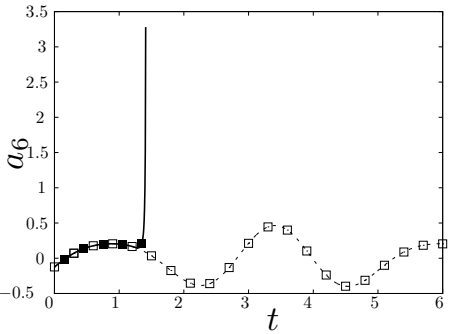

(c) Mode 6

Figure 5. Comparison between the temporal evolutions of the projected $\square$ (POD) and predicted - (POD ROM) mode amplitudes. The POD ROM is calibrated by minimization of $\mathcal{I}^{(3)}$ with determination of all the coefficients (constant, linear and quadratic). The linear system is not regularized.

\section{APPLICATION TO THE CYLINDER WAKE FLOW}

The methods of calibration presented in section 3 are applied to the cylinder wake flow of section 2.3. In section 4.1, the coefficients of the model are found by first minimizing $\mathcal{I}^{(1)}$ under the constraint of $\mathcal{P}_{C}$, and then by minimizing $\mathcal{I}^{(3)}$. In the subsequent section 4.2 , the results determined by minimizing $\mathcal{J}_{\alpha}^{(2)}$ and $\mathcal{J}_{\alpha}^{(3)}$ are presented. The choice of the weighting parameter $\alpha$ and its impact on the POD ROM are discussed.

\subsection{Minimization of $\mathcal{I}^{(1)}$ under the constraint of $\mathcal{P}_{C}$ and minimization of $\mathcal{I}^{(3)}$}

In Table I, the normalized errors and costs of the calibration are reported for the minimization of $\mathcal{I}^{(1)}$, under the constraint of $\mathcal{P}_{C}$, and for the minimization of $\mathcal{I}^{(3)}$. In that case, different control parameters are considered: determination of the linear, constant and linear, eddy viscosity terms of the model. The results are compared with those obtained by the intrinsic stabilization scheme recently suggested in [7].

It is found that the most effective method of calibration in terms of reductions of normalized errors corresponds to the minimization of $\mathcal{I}^{(1)}$ under the constraint of the Cauchy problem $\mathcal{P}_{C}$, for any specific criterion $\mathcal{E}^{(i)}$ considered. For the minimization of $\mathcal{I}^{(3)}$, the results confirm the intuitive idea that the normalized error decreases as the number of calibration variables increases. Indeed, for this method of calibration, the lowest normalized error is systematically obtained for the determination of constant and linear coefficients. For the corresponding costs of calibration, it is found that for the minimization of $\mathcal{I}^{(1)}$ under the constraint of $\mathcal{P}_{C}, \sqrt{\mathcal{D}}$ is approximately equal to $15 \%$ whereas the cost is only of $3 \%$ in the case of the minimization of $\mathcal{I}^{(3)}$. Since the normalized errors are of the same order of magnitude in both methods, we are tempted to claim that the minimization of $\mathcal{I}^{(3)}$, with the determination of the constant and linear coefficients, is more effective than the other approaches of calibration. However, the economic argument (ratio of savings over costs) commonly used in flow control to assess the efficiency of control strategies, makes here little sense, because the main objective of the calibration is to determine POD ROMs of improved accuracy. For the methods based on identification of parameters, the cost corresponds to the numerical implementation of the 
calibration and not to the variation of the coefficients from their value determined by POD Galerkin, as the conclusions drawn by [3] may let it think. The cost of calibration, as it is defined by $\mathcal{D}$, is interesting, but especially as an indication, as it is difficult in practice to use this criterion to determine the method of calibration to apply (see section 4.2). Lastly, concerning the intrinsic stabilization scheme, one notes that, on this flow configuration, it is always less effective in terms of normalized errors than other methods.

Since the minimization of $\mathcal{I}^{(1)}$, under the constraint of $\mathcal{P}_{C}$, and the minimization of $\mathcal{I}^{(3)}$ with determination of the constant and linear coefficients seem both as effective, we have tried to use the calibration based on minimization of $\mathcal{I}^{(3)}$ to determine all the coefficients. Figure 5 shows that the POD ROM calibrated in this way diverges quickly during the numerical integration. This behavior, which can seem surprising for this simple kind of dynamics, will be explained in section 4.2 by the ill-conditioning of $A_{0}^{(3)}$ (see Fig. 8).
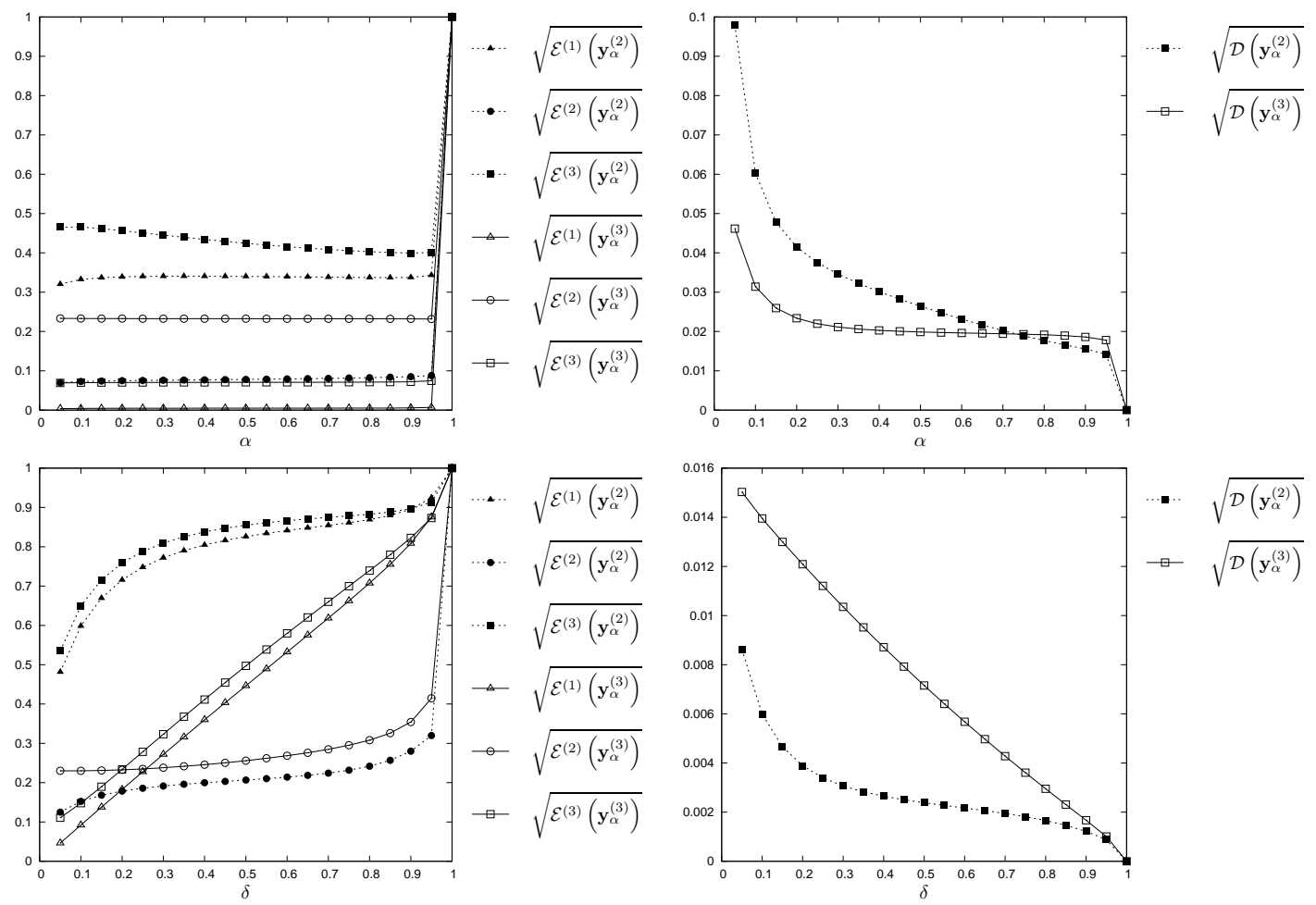

Figure 6. Normalized errors $\mathcal{E}^{(i)}$ and costs of the calibration $\mathcal{D}$ obtained using the minimizations of $\mathcal{J}_{\alpha}^{(2)}$ and $\mathcal{J}_{\alpha}^{(3)}$, for $\alpha$ varying in $\{0.05,0.1, \cdots, 1\}$ (top) and $\delta$ varying in $\{0.05,0.1, \cdots, 1\}$ (bottom).

\subsection{Minimizations of $\mathcal{J}_{\alpha}^{(2)}$ and $\mathcal{J}_{\alpha}^{(3)}$}

In this section, the calibration is carried out by minimizing the functionals $\mathcal{J}_{\alpha}^{(2)}$ and $\mathcal{J}_{\alpha}^{(3)}$ suggested in [3]. Their idea was to determine the coefficients of calibration as solutions of 
an optimization problem aiming at minimizing a weighted average of the normalized error, and a term measuring the variation of the coefficients from their value obtained by POD Galerkin. The main difficulty is to evaluate efficiently, and possibly using an optimal procedure, the value of the weighting parameter $\alpha$. Figure 6 (top) represents the evolutions of the normalized errors and the calibrations costs obtained by minimizing $\mathcal{J}_{\alpha}^{(2)}$ and $\mathcal{J}_{\alpha}^{(3)}$ as $\alpha$ is varied $(\alpha \in\{0.05,0.1, \cdots, 1\})$. Note that by definition of the functional $\mathcal{J}_{\alpha}^{(i)}$, only $\mathcal{E}^{(2)}\left(\mathbf{y}_{\alpha}^{(2)}\right)$, $\mathcal{E}^{(3)}\left(\mathbf{y}_{\alpha}^{(3)}\right), \mathcal{D}\left(\mathbf{y}_{\alpha}^{(2)}\right)$ and $\mathcal{D}\left(\mathbf{y}_{\alpha}^{(3)}\right)$ are monotone functions of $\alpha$. In addition, since the curves of errors and costs vary extremely rapidly when $\alpha$ tends to 1 , we have made the same choice as in [3], and introduced also a variation of these variables according to a parameter $\delta$ defined in increasing bijection with $\alpha$ on $[0,1]$. The weighting parameter $\alpha$ is defined by:

$$
\alpha=\frac{\delta}{\zeta^{(i)}(1-\delta)+\delta} \quad \text { with } \quad \zeta^{(i)}=\frac{\mathcal{I}^{(i)}\left(\mathbf{y}^{G P}\right)}{\mathcal{I}^{(i)}(\mathbf{0})}
$$

and is displayed as a function of $\delta$ in Fig. 7, with $\delta \in\{0.05,0.1, \cdots, 1\}$. The results computed with a linear variation of $\delta$ on $[0,1]$ are represented in Fig. 6 (bottom). Numerically, when $\alpha$ tends to 0 , the trend tends towards a decrease of the normalized error (better calibrated model), and towards an increase of the cost of calibration (model more modified). In practice, it is thus difficult to exploit these curves to choose the value of the appropriate weighting parameter $\alpha$. Of course, it is always possible to arbitrarily choose a criterion, ensuring a specific balance between the reduction of the normalized error and the cost of calibration. However, as it has already been discussed in the previous section, the cost of the calibration measured by the criterion $\mathcal{D}$ is not fully relevant. Then, we may wonder if it would not be more convenient to simply minimize the normalized error by setting $\alpha=0$. The answer is given in Fig. 8, by showing that matrices $A_{\alpha}^{(2)}$ and $A_{\alpha}^{(3)}$ become very ill-conditioned as $\alpha$ goes to 0 . The suggestion of [3] to add the term of calibration cost $\mathcal{D}$ to the functional can be interpreted as a particular method of regularization of the matrix $A^{(i)}$, which is, by nature, ill-conditioned. Moreover, the drawback of the procedure is the choice of the weighting parameter $\alpha$ which is not straightforward. In addition, minimizing $\mathcal{J}_{\alpha}^{(2)}$ and $\mathcal{J}_{\alpha}^{(3)}$ is impossible when coefficients resulting from Galerkin projection are not available (case of the majority of the experimental data). In the next section, we thus propose another method of regularization directly based on the minimization of $\mathcal{I}^{(3)}$.

\section{CALIBRATION BY TIKHONOV REGULARIZATION}

\subsection{Filter factors and discrete Picard condition}

The minimization of the functional $\mathcal{I}^{(3)}$ amounts to solving the linear system $A^{(3)} \mathbf{y}=\mathbf{b}^{(3)}$ where $A^{(3)}$ and $\mathbf{b}^{(3)}$ are given in Appendix III. For the sake of clarity, the superscript is omitted and we thus write simply the linear system as $A \mathbf{y}=\mathbf{b}$. In practice, the right-hand side is contaminated by approximation errors related to the numerical evaluation of the timederivatives of the POD eigenfunctions (remind that $\mathbf{e}^{(3)}(\mathbf{0}, t)=\dot{\mathbf{a}}^{P}(t)$ ). To understand the influence of these errors on the solution of the linear system, the concept of filter factors is 


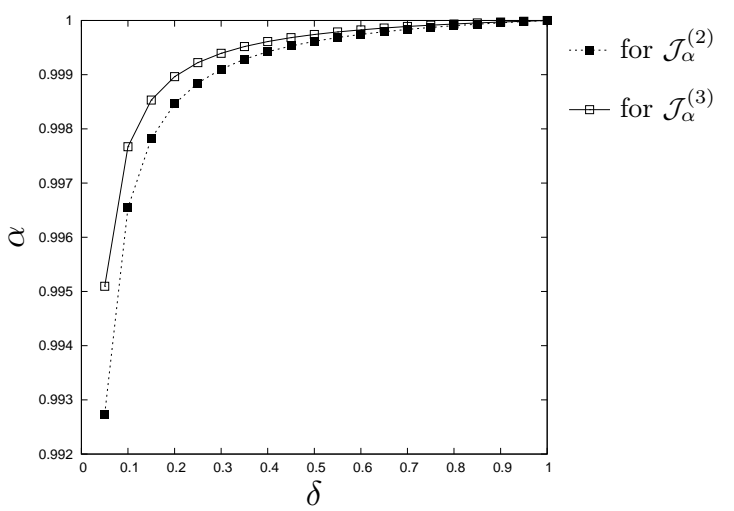

Figure 7. Evolution of $\alpha$ with respect to $\delta$.
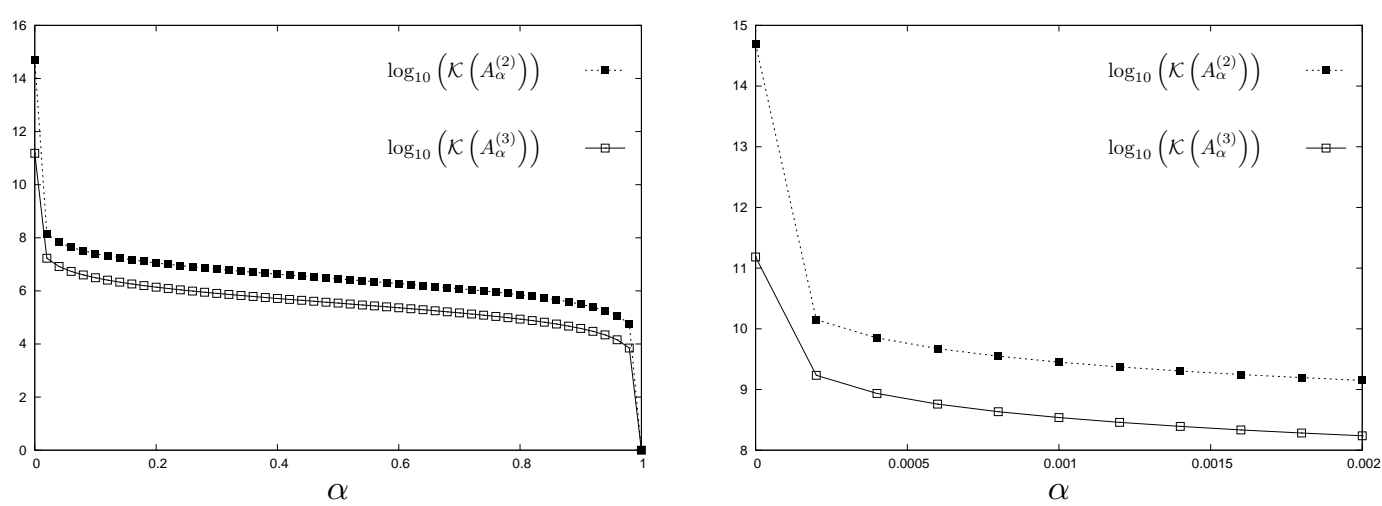

Figure 8. Condition numbers $\mathcal{K}\left(A_{\alpha}^{(i)}\right)(i=2,3)$ obtained during the minimizations of $\mathcal{J}_{\alpha}^{(2)}$ and $\mathcal{J}_{\alpha}^{(3)}$, with $\alpha$ varying in $\{0 ., 0.002, \cdots, 1\}$ (left) and $\alpha$ varying in $\{0 ., 0.0002, \cdots, 0.002\}$ (right).

introduced. To do so, the Singular Value Decomposition [24] is applied to the matrix $A$ :

$$
A=U \Sigma V^{T}=\sum_{j=1}^{N_{\mathbf{y}}} \mathbf{u}_{j} \sigma_{j} \mathbf{v}_{j}^{T},
$$

where $U=\left(\mathbf{u}_{1}, \cdots, \mathbf{u}_{N_{\mathbf{y}}}\right)$ and $V=\left(\mathbf{v}_{1}, \cdots, \mathbf{v}_{N \mathbf{y}}\right)$ are orthogonal matrices containing the left $\mathbf{u}_{j}$ and right $\mathbf{v}_{j}$ singular vectors, and where $\Sigma=\operatorname{diag}\left(\sigma_{1}, \cdots, \sigma_{N_{\mathbf{y}}}\right)$ is a diagonal matrix with the singular values $\sigma_{j}$ arranged in non-increasing order such that

$$
\sigma_{1} \geq \cdots \geq \sigma_{N_{\mathbf{y}}} \geq 0
$$

Since $U$ and $V$ are orthogonal matrices $\left(U U^{T}=V V^{T}=I_{N_{\mathbf{y}}}\right)$, the solution $\mathbf{y}$ of the linear 


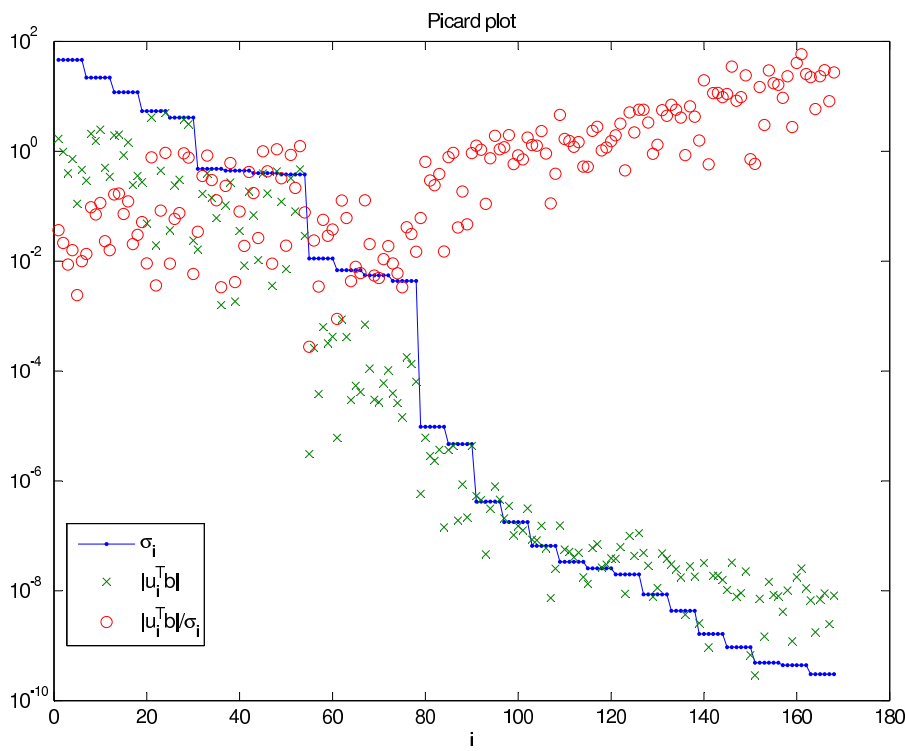

Figure 9. Visual check of the discrete Picard condition corresponding to the minimization of $\mathcal{I}^{(3)}$ with determination of all the coefficients (constant, linear and quadratic) and application of the Tikhonov regularization $\left(L=I ; \mathbf{y}_{0}=\mathbf{y}^{G P}\right)$.

system is given by ${ }^{\ddagger}$ :

$$
\mathbf{y}=\sum_{j=1}^{N_{\mathbf{y}}} \frac{1}{\sigma_{j}} \mathbf{u}_{j}^{T} \mathbf{b} \mathbf{v}_{j}=\sum_{j=1}^{N_{\mathbf{y}}} h_{j} \frac{1}{\sigma_{j}} \mathbf{u}_{j}^{T} \mathbf{b} \mathbf{v}_{j} \quad \text { with } \quad h_{j}=1 \quad \text { for } \quad j=1, \cdots, N_{\mathbf{y}},
$$

where $h_{j}$ are the filter factors. This relation clearly illustrates the numerical difficulties encountered when the linear system is solved without precautions. Indeed, if the Fourier coefficients $\left|\mathbf{u}_{j}^{T} \mathbf{b}\right|$, corresponding to the smaller singular values $\sigma_{j}$, do not decrease sufficiently fast compared to the singular values, the solution is dominated by the terms in the sum corresponding to the smallest $\sigma_{j}$. This behavior can be assessed by inspecting the discrete Picard condition plotted in Fig. 9: for $j \simeq 80$, the singular values decay faster than the Fourier coefficients $\left|\mathbf{u}_{j}^{T} \mathbf{b}\right|$. As a result, the solution obtained presents many oscillations around zero, and thus appears to be completely random (see Fig. 10 for the solution without regularization). To fix this, the first idea is to modify the filter factor $h_{j}$ so that it behaves like an ideal low-pass

${ }^{\ddagger}$ It can be shown easily (see Appendix III) that $\mathbf{y}$ is also the solution of the linear least-squares problem

$$
\min _{\mathbf{y}}\|A \mathbf{y}-\mathbf{b}\|_{2}^{2} \text {. }
$$




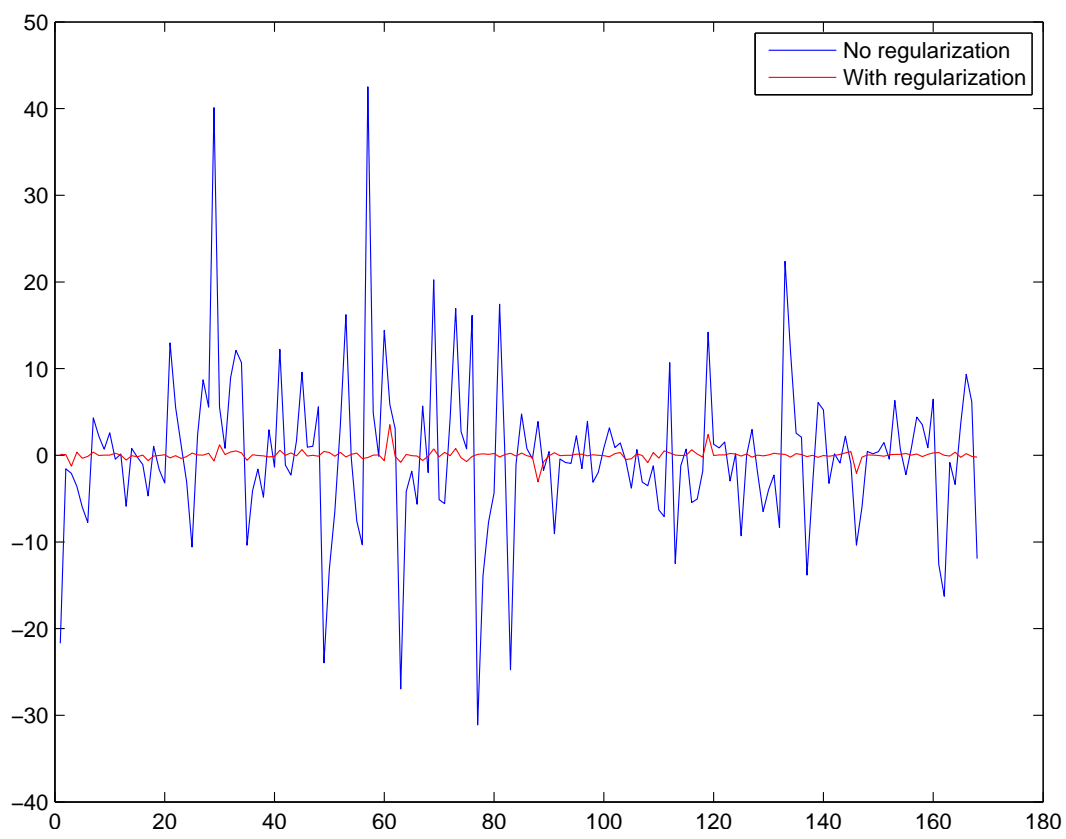

Figure 10. Comparison between solutions obtained with and without regularization, for the minimization of $\mathcal{I}^{(3)}$, with the determination of all the coefficients (constant, linear and quadratic). The linear system is regularized by Tikhonov with $L=I$ and $\mathbf{y}_{0}=\mathbf{y}^{G P}$. Note that for $N_{\text {gal }}=6$, $N_{\mathbf{y}}=168$.

filter:

$$
h_{j}= \begin{cases}1 & \text { if } j \leq 80 \\ 0 & \text { if } j>80\end{cases}
$$

For the cylinder wake flow, this procedure may be appropriate since the temporal dynamics is relatively simple (see the abrupt falling-off in the singular values for $j \simeq 80$ ). However, this method is not suitable for more complex dynamics (3-D turbulent flow for instance) for which the singular values decrease continuously. It is thus necessary to modify the filter factors in a more sophisticated way.

\subsection{Tikhonov regularization}

Undoubtedly, the most common and well-known method of regularization is the Tikhonov regularization [25]. The idea is to seek the regularized solution $\mathbf{y}_{\rho}$ as the minimizer of the following weighted functional

$$
\Phi_{\rho}(\mathbf{y})=\|A \mathbf{y}-\mathbf{b}\|_{2}^{2}+\rho\left\|L\left(\mathbf{y}-\mathbf{y}_{0}\right)\right\|_{2}^{2},
$$

where the first term corresponds to the residual norm, and the second to a side constraint imposed on the solution. $\rho$ is called the regularization parameter and $L$ represents the discrete approximation matrix of a differential operator. This matrix is typically either the identity 
matrix of order $N_{\mathbf{y}}$ (derivative of order zero), or a banded matrix of dimension $\left(N_{\mathbf{y}}-d\right) \times N_{\mathbf{y}}$ approximation of the derivative operator of order $d$. In particular, for $d=0, d=1$ and $d=2$, the method is termed zeroth, first and second-order Tikhonov regularization respectively. Thereafter, these operators will be denoted $L=I(d=0), L=F O D(d=1)$ and $L=S O D$ $(d=2)$.

Intuitively, the regularization can be seen as a balance between two requirements:

1. $\mathbf{y}_{\rho}$ should give a small residual $A \mathbf{y}_{\rho}-\mathbf{b}$,

2. $L\left(\mathbf{y}_{\rho}-\mathbf{y}_{0}\right)$ should be small with respect to the 2 -norm.

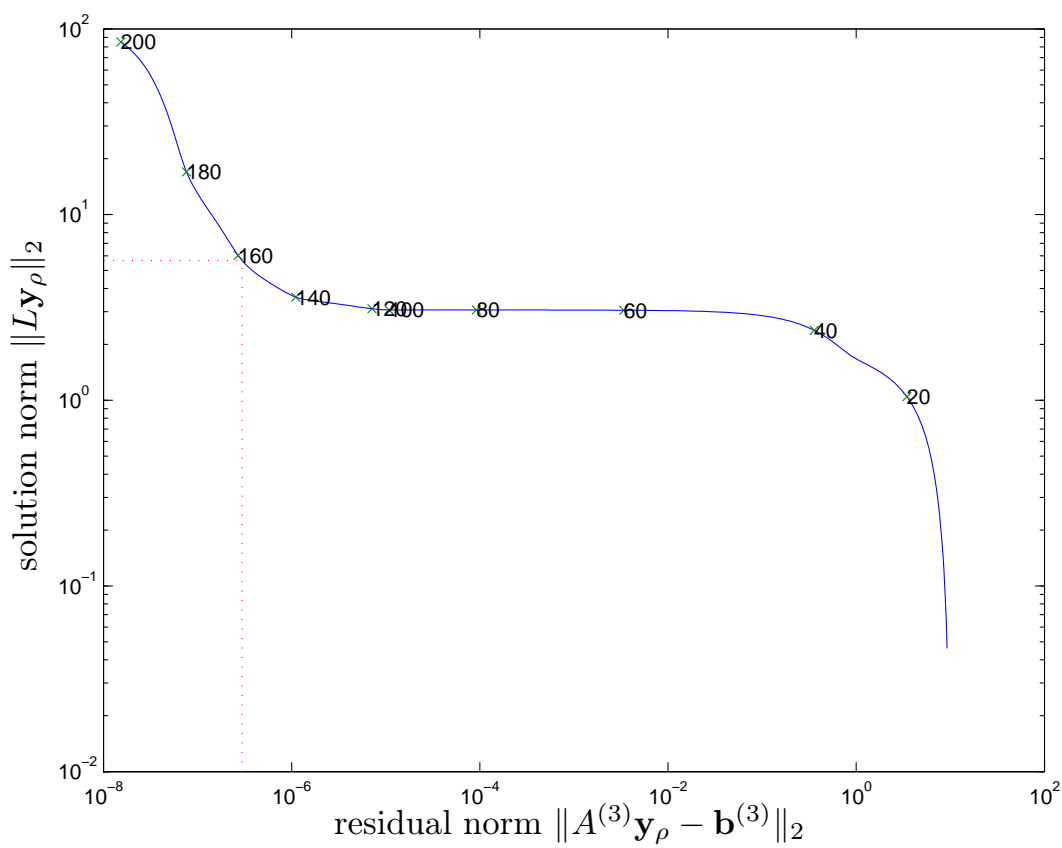

Figure 11. L-curve corresponding to the minimization of $\mathcal{I}^{(3)}$, with the determination of all the coefficients (constant, linear and quadratic) and application of the Tikhonov regularization $(L=I$; $\left.\mathbf{y}_{0}=\mathbf{y}^{G P}\right)$. The "corner" of the L-curve is at $\rho=6.7810^{-8}$.

By using the same type of argument that that of section 5.1 to justify the introduction of filter factors $h_{j}$, it becomes possible to prove that for $\mathbf{y}_{0}=\mathbf{0}$, the regularized solution $\mathbf{y}_{\rho}$ can be written as follows:

$$
\mathbf{y}_{\rho}=\sum_{j=1}^{N_{\mathbf{y}}} h_{j} \frac{1}{\sigma_{j}} \mathbf{u}_{j}^{T} \mathbf{b v}_{j} \quad \text { with } h_{j}=\frac{\sigma_{j}^{2}}{\sigma_{j}^{2}+\rho} \quad \text { if } \quad L=I_{N_{\mathbf{y}}}
$$

and

$$
\mathbf{y}_{\rho}=\sum_{j=1}^{N_{\mathbf{y}}-d} h_{j} \frac{1}{\sigma_{j}} \mathbf{u}_{j}^{T} \mathbf{b} \mathbf{x}_{j}+\sum_{j=N_{\mathbf{y}}-d+1}^{N_{\mathbf{y}}} \mathbf{u}_{j}^{T} \mathbf{b} \mathbf{x}_{j} \quad \text { with } h_{j}=\frac{\gamma_{j}^{2}}{\gamma_{j}^{2}+\rho} \quad \text { if } \quad L \neq I_{N_{\mathbf{y}}} .
$$


Here $\gamma_{j}\left(j=1, \cdots, N_{\mathbf{y}}-d\right)$ are the generalized singular values of $(A, L)$ and $\mathbf{x}_{j}$ the $j$ th column of $X \in \mathbb{R}^{N_{\mathbf{y}} \times N_{\mathbf{y}}}$ (see Appendix $\mathrm{V}$ for the definition of the Generalized Singular Value Decomposition).

The regularization parameter $\rho$ needs now to be computed. To do so, the L-curve method implemented in the package REgularization Tools [26] is used throughout the paper. The L-curve method is based on the analysis of the curve representing the semi-norm of the regularized solution $\left\|L \mathbf{y}_{\rho}\right\|_{2}$, versus the corresponding residual norm $\left\|A \mathbf{y}_{\rho}-\mathbf{b}\right\|_{2}$. In most of the cases, this curve exhibits a typical L shape (see Fig. 11). The corner of the L-curve represents a fair compromise between the minimization of the norm of the residual (horizontal branch) and the semi-norm of the solution (vertical branch). In [26], the detection of the corner is based on the maximization of the curvature of the L-curve.

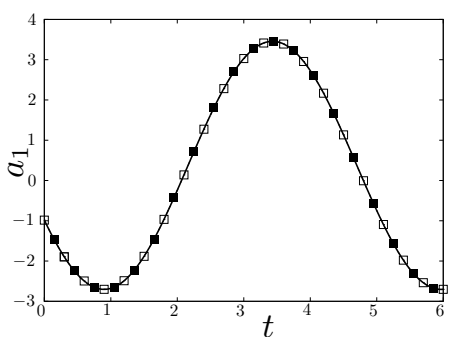

(a) Mode 1

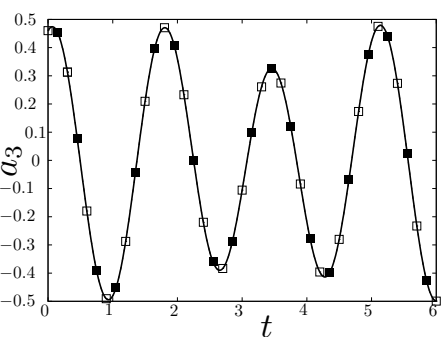

(b) Mode 3

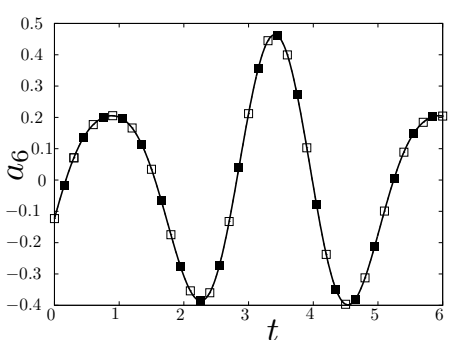

(c) Mode 6

Figure 12. Comparison between the temporal evolutions of the projected $\square$ (POD) and predicted (POD ROM) mode amplitudes. The POD ROM is calibrated by minimizing $\mathcal{I}^{(3)}$, with the determination of all the coefficients (constant, linear and quadratic). The linear system is regularized by Tikhonov with $L=I$ and $\mathbf{y}_{0}=\mathbf{y}^{G P}$.

\subsection{Comparison of the different types of Tikhonov regularization}

Table II. Normalized errors $\mathcal{E}^{(i)}$ and costs of the calibration $\mathcal{D}$. Comparison between the results obtained by minimizing $\mathcal{I}^{(3)}$, with the determination of all the coefficients (constant, linear and quadratic), for different types of Tikhonov regularization. The case $L=F O D$ is not reported because the numerical integration of the calibrated model is diverging.

\begin{tabular}{|l|c|c|c||c|}
\hline Type of Tikhonov regularization & $\sqrt{\mathcal{E}^{(1)(\mathbf{y})}}$ & $\sqrt{\mathcal{E}^{(2)}(\mathbf{y})}$ & $\sqrt{\mathcal{E}^{(3)}(\mathbf{y})}$ & $\sqrt{\mathcal{D}(\mathbf{y})}$ \\
\hline$L=I ; \mathbf{y}_{0}=\mathbf{0}$ & $4.1410^{-3}$ & $2.3310^{-1}$ & $6.2710^{-2}$ & $8.9010^{-1}$ \\
$L=I ; \mathbf{y}_{0}=\mathbf{y}^{G P}$ & $2.3310^{-3}$ & $2.3310^{-1}$ & $6.3110^{-2}$ & $5.6710^{-1}$ \\
$L=S O D ; \mathbf{y}_{0}=\mathbf{0}$ & $2.2710^{-1}$ & $2.2810^{-1}$ & $1.1410^{-1}$ & 1.22 \\
$L=S O D ; \mathbf{y}_{0}=\mathbf{y}^{G P}$ & $5.2110^{-3}$ & $2.3210^{-1}$ & $7.0710^{-2}$ & $3.4410^{-2}$ \\
\hline
\end{tabular}

In this section, the various types of Tikhonov regularization introduced in section 5.2 are compared on the configuration of the cylinder wake flow. Table II reports the normalized errors 


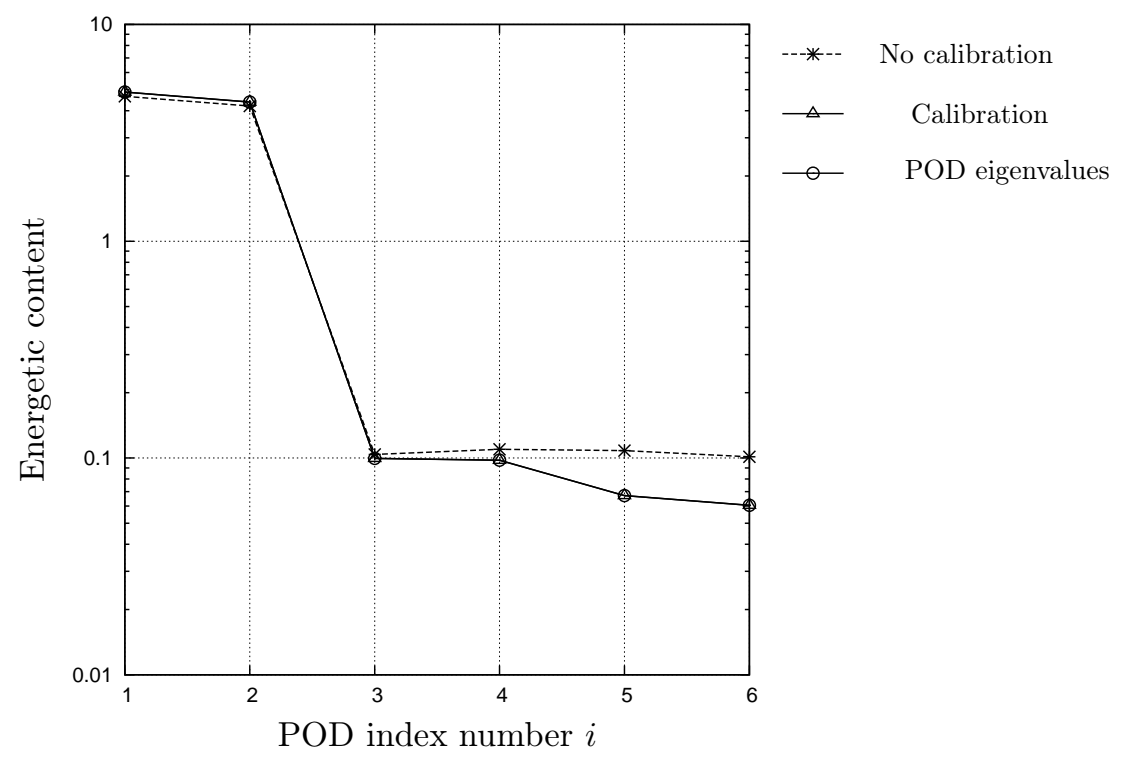

Figure 13. Comparison between the modal energetic contents obtained before and after calibration. The POD eigenvalues are reported for reference. The POD ROM is calibrated by minimizing $\mathcal{I}^{(3)}$, with the determination of all the coefficients (constant, linear and quadratic). The linear system is regularized by Tikhonov with $L=I$ and $\mathbf{y}_{0}=\mathbf{y}^{G P}$.

and costs of the calibration obtained by zeroth and second-order Tikhonov regularization. The case of first-order Tikhonov regularization $(L=F O D)$ is not mentioned because it is diverging, despite the regularization carried out. On average over all the criteria of normalized errors, the case $L=I$ and $\mathbf{y}_{0}=\mathbf{y}^{G P}$ appears to be the most effective. Consequently, this type of Tikhonov regularization is retained for the comparison of section 5.4. In Fig. 12, the temporal evolutions of the POD modes are compared with those predicted by the calibrated reducedorder model. Contrary to the results presented in Fig. 4 there is no clear difference in the dynamics. The immediate consequence is that the modal energy distribution associated to the calibrated model now corresponds perfectly to the POD energy (see figure 13). However, it is worth mentioning that the finite-time thermodynamics (FTT) formalism recently suggested in [27] is more satisfactory because the calibration techniques are rather a posteriori methods whereas the FTT is a flow modeling approach in itself.

\subsection{Comparison of the most effective calibration methods}

To conclude, we compare in this section the three most effective methods of calibration presented in this paper on the configuration of the wake flow. Table III gives the normalized errors and the costs of the calibration obtained by minimization of $\mathcal{I}^{(1)}$ under the constraint of $\mathcal{P}_{C}$, by minimization of $\mathcal{J}_{\alpha}^{(3)}$ for $\alpha=0.001$ and by minimization of $\mathcal{I}^{(3)}$ with determination of all the coefficients of the model and application of the most effective Tikhonov regularization i.e. $L=I$ and $\mathbf{y}_{0}=\mathbf{y}^{G P}$. Except for the $\mathcal{E}^{(3)}$ criterion where $\sqrt{\mathcal{E}^{(3)}(\mathbf{y})}=6.2910^{-2}$ for the 
minimization of $\mathcal{J}_{\alpha}^{(3)}$ and $\sqrt{\mathcal{E}^{(3)}(\mathbf{y})}=6.3110^{-2}$ for the minimization of $\mathcal{I}^{(3)}$ with Tikhonov regularization, the numerical experiments prove that the normalized errors are minimized by the calibration based on Tikhonov regularization. The difference between these methods of calibration can be analyzed in a finer way by introducing the modal errors $\mathcal{I}_{i}^{(j)} \operatorname{defined}^{\S}$ for $i=1, \cdots, N_{\text {gal }}$ and $j=1, \cdots, 3$ as:

$$
\mathcal{I}^{(j)}(\mathbf{y})=\sum_{i=1}^{N_{\text {gal }}} \mathcal{I}_{i}^{(j)}(\mathbf{y}) .
$$

The modal errors $\mathcal{I}_{i}^{(1)}$ are represented in Fig. 14 for the various calibration techniques presented in Table III. For all POD modes, the minimization of $\mathcal{I}^{(3)}$ using the Tikhonov regularization is more effective than the minimization of $\mathcal{J}_{\alpha}^{(3)}$ for $\alpha=0.001$. Additionally, these methods clearly outperform the minimization of $\mathcal{I}^{(1)}$ under the constraint of $\mathcal{P}_{C}$ for the higher POD modes. The main interest of the calibration technique based on the Tikhonov regularization is that the choice of the parameter of regularization $\rho$ is determined by the L-curve without any intervention of the user.

Table III. Normalized errors $\mathcal{E}^{(i)}$ and costs of the calibration $\mathcal{D}$. Comparison between the results obtained by: $i$ ) minimizing $\mathcal{I}^{(1)}$ under the constraint of $\left.\mathcal{P}_{C}, i i\right)$ minimizing $\mathcal{J}_{\alpha}^{(3)}$ with $\alpha=0.001$, and iii) minimizing $\mathcal{I}^{(3)}$ with the determination of all the coefficients (constant, linear and quadratic) and application of the Tikhonov regularization $\left(L=I ; \mathbf{y}_{0}=\mathbf{y}^{G P}\right)$.

\begin{tabular}{|l|c|c|c|c||c|}
\hline Method of calibration & Control terms & $\sqrt{\mathcal{E}^{(1)(\mathbf{y})}}$ & $\sqrt{\mathcal{E}^{(2)}(\mathbf{y})}$ & $\sqrt{\mathcal{E}^{(3)}(\mathbf{y})}$ & $\sqrt{\mathcal{D}(\mathbf{y})}$ \\
\hline Minimization of $\mathcal{I}^{(1)}$ & $C$ and $L$ & $2.6010^{-2}$ & $2.4310^{-1}$ & $2.4410^{-1}$ & $1.5310^{-1}$ \\
under the constraint of $\mathcal{P}_{C}$ & & & & & \\
Minimization of $\mathcal{J}_{\alpha}^{(3)}(\alpha=0.001)$ & $C, L$ and $Q$ & $3.0110^{-3}$ & $2.3310^{-1}$ & $6.2910^{-2}$ & $5.0510^{-1}$ \\
Minimization of $\mathcal{I}^{(3)}$ with Tikhonov & $C, L$ and $Q$ & $2.3310^{-3}$ & $2.3310^{-1}$ & $6.3110^{-2}$ & $5.6710^{-1}$ \\
regularization $\left(L=I ; \mathbf{y}_{0}=\mathbf{y}^{G P}\right)$ & & & & & \\
\hline
\end{tabular}

\section{CONCLUSIONS AND OUTLOOK}

In the first part of this paper, we have presented within a unified framework the various methods of calibration used so far in the literature to identify the coefficients of the POD ROM. Afterwards, we have applied these methods to a 2-D cylinder wake flow, to understand these techniques in details, and, if possible, to release a more effective strategy. We have thus showed that the minimization of $\mathcal{I}^{(1)}$, under the constraint of the Cauchy problem $\mathcal{P}_{C}$, is much more effective than the methods based on the minimization of $\mathcal{I}^{(3)}$, or than the Intrinsic Stabilization scheme. We have then continued by applying the procedure suggested in [3],

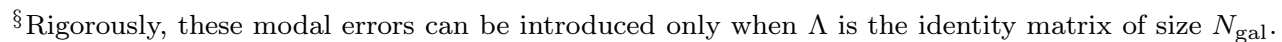




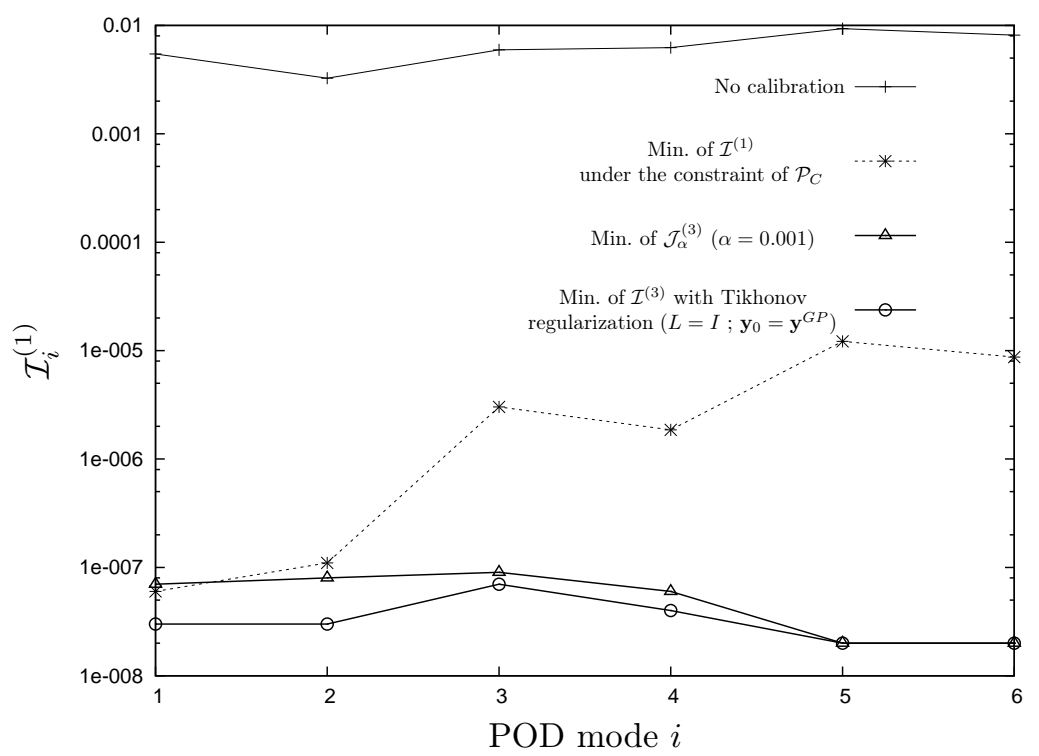

Figure 14. Modal errors $\mathcal{I}_{i}^{(1)}$. Comparison between the results obtained by: $i$ ) minimization of $\mathcal{I}^{(1)}$ under the constraint of $\left.\mathcal{P}_{C}, i i\right)$ minimization of $\mathcal{J}_{\alpha}^{(3)}$ with $\alpha=0.001$ and iii) minimization of $\mathcal{I}^{(3)}$ with determination of all the coefficients (constant, linear and quadratic) and application of the Tikhonov regularization $\left(L=I ; \mathbf{y}_{0}=\mathbf{y}^{G P}\right)$. We remind that $\mathcal{I}^{(1)}(\mathbf{y})=\sum_{i=1}^{N_{\text {gal }}} \mathcal{I}_{i}^{(1)}(\mathbf{y})$.

which is based on the minimization of a weighted sum of the normalized error, and of a term measuring the variation of the coefficients of the model to their values obtained by POD Galerkin. In substance, the idea is identical to what is usually made in optimal control ([28] for instance). Indeed, the cost functional is built as the sum of two terms, the first being a measure of the objective of the optimization, and the second corresponding to the cost of the control. However, as far as the identification is concerned, the variation of the coefficients of the calibrated model from their values obtained by POD Galerkin, is not relevant because the main objective is to improve the accuracy of the model. Also, in the case of experimental data, the coefficients resulting from POD Galerkin do not even exist in the majority of the cases. Finally, it can be shown numerically that adding the cost of the calibration to the functional to be minimized, comes down to improve the conditioning of the linear systems associated to the minimization of $\mathcal{I}^{(2)}$ and $\mathcal{I}^{(3)}$. As the choice of the parameter $\alpha$ is relatively tricky, even arbitrary, the linear system associated to the minimization of $\mathcal{I}^{(3)}$ is regularized by the method of Tikhonov. For our test-case, the zeroth-order regularization $(L=I)$ with $\mathbf{y}_{0}=\mathbf{y}^{G P}$ is the most effective among all the types of Tikhonov regularization considered. Lastly, our numerical experiments demonstrate that the Tikhonov regularization outperforms, in terms of normalized errors, the minimization of $\mathcal{I}^{(1)}$ under the constraint of $\mathcal{P}_{C}$ and the minimization of $\mathcal{J}_{\alpha}^{(3)}$ with $\alpha=0.001$. Compared to the approach suggested in [3], the interest of the Tikhonov regularization is that the value of the parameter of regularization $\rho$ is determined automatically, via the L-curve method. 
The calibration by Tikhonov regularization presented here still needs to be tested on flow configurations corresponding to more complex dynamics: 3-D turbulent flow obtained by numerical simulations, and challenging experimental data. The extension to actuated configurations with known control parameters should be straightforward. On the other hand, calibrating a reduced-order model, so that it can represent the evolution of dynamics when the control law varies, is a more complex task. A basic idea consists in determining a POD basis starting from snapshots coming from several control laws, and then to calibrate the model for all the control laws considered. Recently, this approach was applied for the first time in [1] to identify a robust model of actuated wakes.

\section{APPENDIX}

\section{POD ROM COEFFICIENTS}

The coefficients of the POD ROM (3) obtained by Galerkin projection (GP) are:

$$
\begin{aligned}
& A_{i}^{G P}=-\left(\boldsymbol{\Phi}_{i},\left(\mathbf{u}_{m} \cdot \nabla\right) \mathbf{u}_{m}\right)_{\Omega}-\frac{1}{R e}\left(\left(\nabla \otimes \boldsymbol{\Phi}_{i}\right)^{T}, \nabla \otimes \mathbf{u}_{m}\right)_{\Omega}+\frac{1}{R e}\left[\left(\nabla \otimes \mathbf{u}_{m}\right) \boldsymbol{\Phi}_{i}\right]_{\partial \Omega}, \\
& B_{i j}^{G P}=-\left(\boldsymbol{\Phi}_{i},\left(\mathbf{u}_{m} \cdot \nabla\right) \boldsymbol{\Phi}_{j}\right)_{\Omega}-\left(\boldsymbol{\Phi}_{i},\left(\boldsymbol{\Phi}_{j} \cdot \nabla\right) \mathbf{u}_{m}\right)_{\Omega}-\frac{1}{R e}\left(\left(\nabla \otimes \boldsymbol{\Phi}_{i}\right)^{T}, \nabla \otimes \boldsymbol{\Phi}_{j}\right)_{\Omega} \\
&+\frac{1}{R e}\left[\left(\nabla \otimes \boldsymbol{\Phi}_{j}\right) \boldsymbol{\Phi}_{i}\right]_{\partial \Omega}, \\
& C_{i j k}^{G P}=-\left(\boldsymbol{\Phi}_{i},\left(\boldsymbol{\Phi}_{j} \cdot \nabla\right) \boldsymbol{\Phi}_{k}\right)_{\Omega}, \\
& D_{i}^{G P}=-\left(\boldsymbol{\Phi}_{i}, \mathbf{u}_{c}\right)_{\Omega}, \\
& E_{i}^{G P}=-\left(\boldsymbol{\Phi}_{i},\left(\mathbf{u}_{c} \cdot \nabla\right) \mathbf{u}_{m}\right)_{\Omega}-\left(\boldsymbol{\Phi}_{i},\left(\mathbf{u}_{m} \cdot \nabla\right) \mathbf{u}_{c}\right)_{\Omega}-\frac{1}{R e}\left(\left(\nabla \otimes \boldsymbol{\Phi}_{i}\right)^{T}, \nabla \otimes \mathbf{u}_{c}\right)_{\Omega} \\
&+\frac{1}{R e}\left[\left(\nabla \otimes \mathbf{u}_{c}\right) \boldsymbol{\Phi}_{i}\right]_{\partial \Omega}, \\
& F_{i j}^{G P}=-\left(\boldsymbol{\Phi}_{i},\left(\boldsymbol{\Phi}_{j} \cdot \nabla\right) \mathbf{u}_{c}\right)_{\Omega}-\left(\boldsymbol{\Phi}_{i},\left(\mathbf{u}_{c} \cdot \nabla\right) \boldsymbol{\Phi}_{j}\right)_{\Omega}, \\
& G_{i}^{G P}=-\left(\boldsymbol{\Phi}_{i},\left(\mathbf{u}_{c} \cdot \nabla\right) \mathbf{u}_{c}\right)_{\Omega}, \\
& \text { with }[\mathbf{u}]_{\partial \Omega}=\int_{\partial \Omega} \mathbf{u} \cdot \mathbf{n} d \mathbf{x} \text { and }(\overline{\bar{P}}, \overline{\bar{Q}})_{\Omega}=\int_{\Omega} \overline{\bar{P}}: \overline{\bar{Q}} d \mathbf{x}=\sum_{i, j=1}^{n_{c}} \int_{\Omega} P_{i j} Q_{j i} d \boldsymbol{x} . \text { Here, } \\
& n_{c} \text { is the number of components of } \mathbf{u} . \text { When the flow is not controlled, } \mathbf{u}_{c}=\mathbf{0} \text { and }
\end{aligned}
$$


$D_{i}^{G P}=E_{i}^{G P}=F_{i j}^{G P}=G_{i}^{G P}=0$. When $\mathcal{U}=\left\{\mathbf{u}^{i}\right\}_{i=1, \ldots, N_{t}}$, we have $\mathbf{u}_{m}=0$ and $A_{i}^{G P}=0$.

\section{EVALUATION OF $\mathbf{f}$ AND g}

The values of $f_{i}$ and $g_{i}$ (see section 2.2.2 for their definitions) can be easily determined if we know the coefficients $\mathbf{y}_{i}$ and $\mathbf{z}_{i}$. Indeed, $f_{i}$ belongs to the space of polynomials of degree 2 in $N_{\text {gal }}$ variables: $a_{1}^{R}(t), \cdots, a_{N_{\text {gal }}}^{R}(t)$. Therefore, let

$$
\mathbf{m}(t)=\left(\begin{array}{c}
1 \\
a_{1}^{R}(t) \\
\vdots \\
a_{N_{\text {gal }}}^{R}(t) \\
a_{1}^{R}(t) a_{1}^{R}(t) \\
\vdots \\
a_{N_{\text {gal }}}^{R}(t) a_{N_{\text {gal }}}^{R}(t)
\end{array}\right) \in \mathbb{R}^{N_{y_{i}}}
$$

be the vector containing the natural monomial basis of this space, we can write:

$$
f_{i}\left(\mathbf{y}_{i}, \mathbf{a}^{R}(t)\right)=\mathbf{m}(t) \cdot \mathbf{y}_{i} .
$$

Similarly, if we define

$$
\mathbf{q}(t)=\left(\begin{array}{c}
\dot{\gamma}(t) \\
\gamma(t) \\
\gamma(t) a_{1}^{R}(t) \\
\vdots \\
\gamma(t) a_{N_{\text {gal }}}^{R}(t) \\
\gamma^{2}(t)
\end{array}\right) \in \mathbb{R}^{N_{z_{i}}}
$$

we have:

$$
g_{i}\left(\mathbf{z}_{i}, \mathbf{a}^{R}(t), \gamma\right)=\mathbf{q}(t) \cdot \mathbf{z}_{i} .
$$

We can deduce from these expressions that $\mathbf{f}$ can be computed at any time instant $t$, as the product of a block diagonal matrix $M$ where each block is equal to $\mathbf{m}^{T}$ by the vector $\mathbf{y}$. Similarly, $\mathbf{g}$ can be obtained as the product of a block diagonal matrix $Q$ where each block is given by $\mathbf{q}^{T}$ by the vector $\mathbf{z}$.

\section{MINIMIZATION OF $\mathcal{I}^{(2)}$ AND $\mathcal{I}^{(3)}$}

For $i=2$ and $3, \mathbf{e}^{(i)}$ is an affine function with respect to $\mathbf{y} \in \mathbb{R}^{N_{\mathbf{y}}}$ (see sections 3.1.2 and 3.1.3). Therefore, we introduce the application

$$
\begin{aligned}
\mathbf{e}^{(i)}(\cdot, t): \mathbb{R}^{N_{\mathbf{y}}} & \rightarrow \mathbb{R}^{N_{\text {gal }}} \\
\mathbf{y} & \mapsto E^{(i)}(t) \mathbf{y}+\mathbf{e}^{(i)}(0, t) \quad \text { with } \quad E^{(i)}(t) \in \mathbb{R}^{N_{\text {gal }} \times N_{\mathbf{y}}} .
\end{aligned}
$$

By identification with the expressions of the errors $\mathbf{e}^{(i)}(i=1,2)$, one finds immediately that: 
- for $i=2$

$$
E^{(2)}(t) \mathbf{y}=-\int_{0}^{t} \mathbf{f}\left(\mathbf{y}, \mathbf{a}^{P}(\tau)\right) d \tau \quad \text { and } \quad \mathbf{e}^{(2)}(\mathbf{0}, t)=\mathbf{a}^{P}(t)-\mathbf{a}^{P}(0)
$$

- for $i=3$

$$
E^{(3)}(t) \mathbf{y}=-\mathbf{f}\left(\mathbf{y}, \mathbf{a}^{P}(t)\right) \quad \text { and } \quad \mathbf{e}^{(3)}(\mathbf{0}, t)=\dot{\mathbf{a}}^{P}(t)
$$

where $\mathbf{f}$ can be evaluated using the method described in Appendix II.

Assuming that $\Lambda$ is symmetric, we can prove that for $i=2$ and 3 :

$$
\begin{aligned}
\mathcal{I}^{(i)}(\mathbf{y})=\left\langle\left\|\mathbf{e}^{(i)}(\mathbf{y}, t)\right\|_{\Lambda}^{2}\right\rangle_{T_{o}}= & \mathbf{y}^{T}\left\langle E^{(i)}(t)^{T} \Lambda E^{(i)}(t)\right\rangle_{T_{o}} \mathbf{y}+2\left\langle\mathbf{e}^{(i)}(0, t)^{T} \Lambda E^{(i)}(t)\right\rangle_{T_{o}} \mathbf{y} \\
& +\left\langle\mathbf{e}^{(i)}(0, t)^{T} \Lambda \mathbf{e}^{(i)}(0, t)\right\rangle_{T_{o}} \\
= & \mathbf{y}^{T} A^{(i)} \mathbf{y}-2 \mathbf{b}^{(i)}{ }^{T} \mathbf{y}+\mathbf{c}^{(i)}
\end{aligned}
$$

where

$$
\begin{gathered}
A^{(i)}=\left\langle E^{(i) T}(t) \Lambda E^{(i)}(t)\right\rangle_{T_{o}} \in \mathbb{R}^{N_{\mathbf{y}} \times N_{\mathbf{y}}}, \\
\mathbf{b}^{(i)}=-\left\langle E^{(i) T}(t) \Lambda \mathbf{e}^{(i)}(0, t)\right\rangle_{T_{o}} \in \mathbb{R}^{N_{\mathbf{y}}}, \\
\mathbf{c}^{(i)}=\left\langle\mathbf{e}^{(i)}(0, t)^{T} \Lambda \mathbf{e}^{(i)}(0, t)\right\rangle_{T_{o}} \in \mathbb{R} .
\end{gathered}
$$

If $\Lambda$ is a symmetric matrix, then $A^{(i)}$ is also symmetric by construction. In that case, minimizing the quadratic function $\mathcal{I}^{(i)}$ is equivalent to solve the linear system defined by:

$$
A^{(i)} \mathbf{y}=\mathbf{b}^{(i)} \text {. }
$$

\section{MINIMIZATION OF $\mathcal{J}_{\alpha}^{(i)}$}

The functional $\mathcal{J}_{\alpha}^{(i)}$ can also be written as:

$$
\mathcal{J}_{\alpha}^{(i)}(\mathbf{y})=\chi_{A}^{\alpha} \underbrace{\mathcal{I}^{(i)}(\mathbf{y})}_{f_{1}(\mathbf{y})}+\chi_{\Pi}^{\alpha} \underbrace{\left\|\mathbf{y}-\mathbf{y}^{G P}\right\|_{\Pi}^{2}}_{f_{2}(\mathbf{y})},
$$

by denoting

$$
\chi_{A}^{\alpha}=\frac{1-\alpha}{\mathcal{I}^{(i)}\left(\mathbf{y}^{G P}\right)} \quad \text { and } \quad \chi_{\Pi}^{\alpha}=\frac{\alpha}{\left\|\mathbf{y}^{G P}\right\|_{\Pi}^{2}} .
$$

In Appendix III, we have demonstrated that when $\Lambda$ is a symmetric matrix we have $f_{1}(\mathbf{y})=\mathbf{y}^{T} A^{(i)} \mathbf{y}-2 \mathbf{b}^{(i)^{T}} \mathbf{y}+\mathbf{c}^{(i)}$. Similarly, it can be shown that $f_{2}(\mathbf{y})=\mathbf{y}^{T} \Pi \mathbf{y}-2 \mathbf{y}^{G P^{T}} \Pi \mathbf{y}+$ $\mathbf{y}^{G P^{T}} \Pi \mathbf{y}^{G P}$ if we assume that $\Pi$ is symmetric.

Since $f_{1}$ and $f_{2}$ are two quadratic functions, it is simple to evaluate their gradients at $\mathbf{y}$. One obtains:

$$
\nabla f_{1}(\mathbf{y})=2\left(A^{(i)} \mathbf{y}-\mathbf{b}^{(i)}\right) \quad \text { and } \quad \nabla f_{2}(\mathbf{y})=2 \Pi\left(\mathbf{y}-\mathbf{y}^{G P}\right)
$$


By definition, the functional $\mathcal{J}_{\alpha}^{(i)}$ is minimal at $\mathbf{y}_{\alpha}^{(i)}$ when $\nabla \mathcal{J}_{\alpha}^{(i)}\left(\mathbf{y}_{\alpha}^{(i)}\right)=0$. Minimizing $\mathcal{J}_{\alpha}^{(i)}(\mathbf{y})$ for $i=2$ or 3 is then equivalent to solve the linear system:

$$
A_{\alpha}^{(i)} \mathbf{y}_{\alpha}^{(i)}=\mathbf{b}_{\alpha}^{(i)},
$$

with

$$
A_{\alpha}^{(i)}=\chi_{A}^{\alpha} A^{(i)}+\chi_{\Pi}^{\alpha} \Pi,
$$

and

$$
\mathbf{b}_{\alpha}^{(i)}=\chi_{A}^{\alpha} \mathbf{b}^{(i)}+\chi_{\Pi}^{\alpha} \Pi \mathbf{y}^{G P} .
$$

Note that for $\alpha=0, \chi_{A}^{\alpha}=\frac{1}{\mathcal{I}^{(i)}\left(\mathbf{y}^{G P}\right)}$ and $\chi_{\Pi}^{\alpha}=0$. Inserting these relations into (7), the linear system (6) is recovered.

\section{GENERALIZED SINGULAR VALUE DECOMPOSITION}

Let $A \in \mathbb{R}^{m \times n}$ and $L \in \mathbb{R}^{p \times n}$ be given with $m \geq n \geq p$. There exist orthogonal matrices $U \in \mathbb{R}^{m \times n}$ and $V \in \mathbb{R}^{p \times p}$ and a nonsingular matrix $X \in \mathbb{R}^{n \times n}$ such that

$$
A=U\left(\begin{array}{cc}
\Sigma & 0 \\
0 & I_{n-p}
\end{array}\right) X^{-1} \quad, \quad L=V(M, 0) X^{-1}
$$

where $\Sigma=\operatorname{diag}\left(\sigma_{1}, \cdots, \sigma_{p}\right)$ and $M=\operatorname{diag}\left(\mu_{1}, \cdots, \mu_{p}\right)$ with $0 \leq \sigma_{1} \leq \cdots \leq \sigma_{p} \leq 1$ and $1 \geq \mu_{1} \geq \cdots \geq \mu_{p} \geq 0$. Furthermore, it holds that $\sigma_{j}^{2}+\mu_{j}^{2}=1$ for $j=1, \cdots, p$. The values $\gamma_{j}=\sigma_{j} / \mu_{j}(j=1, \cdots, p)$ are called the generalized singular values of $(A, L)$. The $j$ th column $x_{j}$ of $X$ is the right singular vector associated with $\sigma_{j}$. 


\section{ACKNOWLEDGEMENTS}

A preliminary version of this work was presented at the workshop on "Industrial applications of low order models based on POD" organized by Angelo Iollo (IMB - Bordeaux University and MC2 INRIA Bordeaux Sud-Ouest). This workshop held at Bordeaux on March 31 - April 2, 2008 was very stimulating and nevertheless friendly. LC is very indebted to Angelo for inviting him to give a talk. The extension of the Tikhonov regularization method to the first and second order presented in this paper was suggested by I. M. Navon (School of Computational Science and Department of Mathematics, Florida State University) during the workshop. LC is very grateful to him for this suggestion. Last but not least, LC would like to acknowledge Bernd R. Noack and the low-dimensional modeling and control team at the Technische Universität Berlin for stimulating and fruitful discussions.

\section{REFERENCES}

1. Weller J, Lombardi E, Iollo A. Robust model identification of actuated vortex wakes. Physica D: Nonlinear Phenomena 2009; 238:416-427.

2. Galletti B, Bruneau CH, Zannetti L, Iollo A. Low-order modelling of laminar flow regimes past a confined square cylinder. J. Fluid Mech. 2004; 503:161-170.

3. Couplet M, Basdevant C, Sagaut P. Calibrated Reduced-Order POD-Galerkin system for fluid flow modelling. J. Comp. Phys. 2005; 207:192-220.

4. Bergmann M, Cordier L, Brancher JP. Optimal rotary control of the cylinder wake using POD Reduced Order Model. Phys. Fluids 2005; 17(9):097 101:1-21.

5. Perret L, Collin E, Delville J. Polynomial identification of POD based low-order dynamical system. Journal of Turbulence 2006; 7:1-15.

6. Galletti B, Bottaro A, Bruneau CH, Iollo A. Accurate model reduction of transient and forced wakes. Eur. J. Mech. B/Fluids 2007; 26(3):354-366.

7. Kalb VL, Deane AE. An intrinsic stabilization scheme for proper orthogonal decomposition based lowdimensional models. Phys. Fluids 2007; 19:054106.

8. Tikhonov AN, Arsenin VY. Solutions of ill posed problems. Winston and Sons, 1977.

9. Alekseev AK, Navon IM. The Analysis of an Ill-Posed Problem Using Multiscale Resolution and Second Order Adjoint Techniques. Computer Methods in Applied Mechanics and Engineering 2001; 190(1517):1937-1953.

10. Rowley CW. Modeling, simulation and control of cavity flow oscillations. Phd thesis, California Institute of Technology 2002.

11. Cordier L, Bergmann M. Proper Orthogonal Decomposition: an overview. Lecture series 2002-04, 200303 and 2008-01 on post-processing of experimental and numerical data. Von Kármán Institute for Fluid Dynamics, 2008.

12. Lumley JL. Atmospheric Turbulence and Wave Propagation. The structure of inhomogeneous turbulence. Nauka, Moscow, 1967; 166-178.

13. Sirovich L. Turbulence and the dynamics of coherent structures. Quarterly of Applied Mathematics 1987; XLV(3):561-590.

14. Noack BR, Afanasiev K, Morzyński M, Tadmor G, Thiele F. A hierarchy of low-dimensional models for the transient and post-transient cylinder wake. J. Fluid Mech. 2003; 497:335-363.

15. Cordier L, Bergmann M. Two typical applications of POD: coherent structures eduction and reduced order modelling. Lecture series 2002-04, 2003-03 and 2008-01 on post-processing of experimental and numerical data. Von Kármán Institute for Fluid Dynamics, 2008.

16. Bergmann M, Cordier L. Optimal control of the cylinder wake in the laminar regime by Trust-Region methods and POD Reduced Order Models. J. Comp. Phys. 2008; 227:7813-7840.

17. Bergmann M, Cordier L. Control of the circular cylinder wake by Trust-Region methods and POD Reduced-Order Models. Research Report 6552, INRIA $062008 . \quad$ URL https://hal.inria.fr/inria-00284258. 
18. Noack BR, Cordier L, Morzyński M, Tadmor G. Reduced Order Models for Analysis and Control of Fluid Flows. Technical Report, Hermann-Föttinger-Institut für Ströungsmechanik, Technische Universität Berlin 2005.

19. Favier J. Contrôle d'écoulements : approche expérimentale et modélisation de dimension réduite. Phd thesis, Institut National Polytechnique de Toulouse, France 2007.

20. Iollo A, Lanteri S, Désidéri JA. Stability properties of POD-Galerkin approximations for the compressible Navier-Stokes equations. Technical Report 3589, INRIA 1998.

21. Rempfer D. On low-dimensional Galerkin models for fluid flow. Theor. Comput. Fluid Dyn. $2000 ; 14: 75-88$.

22. Bergmann M. Optimisation aérodynamique par réduction de modèle POD et contrôle optimal. Application au sillage laminaire d'un cylindre circulaire. Phd thesis, Institut National Polytechnique de Lorraine, Nancy, France 2004

23. Bourguet R, Braza M, Dervieux A. Reduced-order modeling for unsteady transonic flows around an airfoil. Phys. Fluids 2007; 19(11):111 701:1-4.

24. Golub GH, Van Loan CF. Matrix Computations. 3 edn., The Johns Hopkins University Press: Baltimore and London, 1996.

25. Hansen PC. Rank-Deficient and Discrete Ill-Posed Problems: Numerical Aspects of Linear Inversion. SIAM, Philadelphia, 1998.

26. Hansen PC. Regularization Tools: A Matlab package for analysis and solution of discrete ill-posed problems. Numerical Algorithms 1994; 6:1-35.

27. Noack BR, Schlegel M, Ahlborn B, Mutschke G, Morzyński M, Comte P, Tadmor G. A finite-time thermodynamics formalism for unsteady flows. J. Non-Equilib. Thermodyn. 2008; 33(2):103-148.

28. Gunzburger MD. Introduction into mathematical aspects of flow control and optimization. Lecture series 1997-05 on inverse design and optimization methods. Von Kármán Institute for Fluid Dynamics, 1997. 